\title{
Attractive strings and five-branes, skew-holomorphic Jacobi forms and moonshine
}

\author{
Miranda C.N. Cheng, ${ }^{a}$ John F.R. Duncan, ${ }^{b}$ Sarah M. Harrison, ${ }^{c, d}$ Jeffrey A. Harvey, ${ }^{e}$ \\ Shamit Kachru ${ }^{f}$ and Brandon C. Rayhaun ${ }^{f}$ \\ ${ }^{a}$ Institute of Physics and Korteweg-de Vries Institute for Mathematics, University of Amsterdam, \\ Amsterdam, the Netherlands \\ ${ }^{b}$ Department of Mathematics and Computer Science, Emory University, \\ Atlanta, GA 30322, U.S.A. \\ ${ }^{c}$ Center for the Fundamental Laws of Nature, Harvard University, \\ Cambridge, MA 02138, U.S.A. \\ ${ }^{d}$ Department of Mathematics and Statistics and Department of Physics, McGill University, \\ Montreal, QC, Canada \\ ${ }^{e}$ Enrico Fermi Institute and Department of Physics, University of Chicago, \\ Chicago IL 6063\%, U.S.A. \\ ${ }^{f}$ Stanford Institute for Theoretical Physics, Stanford University, \\ Palo Alto, CA 94305, U.S.A. \\ E-mail: chengm@math.jussieu.fr, john.duncan@emory.edu, \\ sarharr@gmail.com, j-harvey@uchicago.edu, skachru@stanford.edu, \\ brandonrayhaun@gmail.com
}

ABSTRACT: We show that certain BPS counting functions for both fundamental strings and strings arising from fivebranes wrapping divisors in Calabi-Yau threefolds naturally give rise to skew-holomorphic Jacobi forms at rational and attractor points in the moduli space of string compactifications. For M5-branes wrapping divisors these are forms of weight negative one, and in the case of multiple M5-branes skew-holomorphic mock Jacobi forms arise. We further find that in simple examples these forms are related to skewholomorphic (mock) Jacobi forms of weight two that play starring roles in moonshine. We discuss examples involving M5-branes on the complex projective plane, del Pezzo surfaces of degree one, and half-K3 surfaces. For del Pezzo surfaces of degree one and certain halfK3 surfaces we find a corresponding graded (virtual) module for the degree twelve Mathieu group. This suggests a more extensive relationship between Mathieu groups and complex surfaces, and a broader role for M5-branes in the theory of Jacobi forms and moonshine.

KeYwords: Black Holes in String Theory, Discrete Symmetries, M-Theory, Superstrings and Heterotic Strings

ARXiv EPrint: 1708.07523 


\section{Contents}

1 Introduction 1

2 Skew-holomorphic Jacobi forms 2

3 Rational heterotic string compactifications 4

3.1 The rational Gaussian model 4

$\begin{array}{ll}3.2 & \text { Heterotic strings with Wilson lines }\end{array}$

$\begin{array}{ll}3.3 & \text { Rational toroidal compactifications }\end{array}$

4 The M5-brane elliptic genus $\quad 10$

$\begin{array}{lll}4.1 \text { Multiplets } & 10\end{array}$

4.2 The index 11

5 Examples $\quad 13$

$\begin{array}{lll}5.1 & \text { The projective plane } & 13\end{array}$

$\begin{array}{lll}5.2 & \text { Degree one del Pezzo surfaces } & 14\end{array}$

$\begin{array}{lll}5.3 & \text { Half-K3 surfaces } & 15\end{array}$

A New Mathieu moonshine 18

\section{Introduction}

Jacobi forms and mock Jacobi forms play important roles as counting functions governing black hole entropy in string theory. For a recent comprehensive discussion see [1]. They also play starring roles in studies of moonshine, as in, e.g., [2-4]. Skew-holomorphic Jacobi forms, first introduced by Skoruppa in [5, 6], also play an important role in moonshine. Indeed, the weight one-half modular forms exhibiting moonshine for the Thompson group in [7] can be recast as the theta components of skew-holomorphic Jacobi forms, an observation extended in [8] to obtain a larger family of moonshine phenomena. In this work we promote the idea that BPS counting functions appearing in the theory of strings and wrapped fivebranes at rational and attractor points provide a rich source of such objects and suggest further new possibilities for connections between moonshine, black holes, and BPS state counting.

Our first main observation is that half-BPS state counting functions for the heterotic string on $S^{1}$ at rational points in the Narain moduli space lead directly to skew-holomorphic Jacobi forms. Our second main observation is that M5-branes wrapping divisors in CalabiYau threefolds, studied in e.g. [9] as giving rise to black strings in M-theory, provide another natural source of skew-holomorphic Jacobi forms. As discussed in [10-13], the modified 
elliptic genera counting supersymmetric states in these theories are non-holomorphic modular forms of a certain kind. We will see that at suitable moduli these functions can be specialized to skew-holomorphic Jacobi forms. A number of examples of such genera were computed in a closely related setup in [14] (note that many of these do not satisfy the "ampleness" assumption of [9]). We will see, in several cases, that a skew-holomorphic Jacobi form or mock Jacobi form of weight 2 which plays a role in moonshine can be extracted. We will focus on cases where either a single M5-brane is wrapped, or two M5branes are wrapped. Skew-holomorphic mock Jacobi forms appear in the latter case, due to the presence of bound states of single wrapped M5-branes.

Another important observation concerns the particular example of a single M5-brane wrapping a del Pezzo surface of degree one (i.e. $\mathbb{P}^{2}$ blown up at eight points). As we explain in section 5.2, the corresponding skew-holomorphic Jacobi form of weight 2 admits an interpretation as a generating function for the graded dimension of a graded virtual module for the sporadic simple group $M_{12}$. This suggests a non-trivial relationship between $M_{12}$ and del Pezzo surfaces, and a concrete path to begin its exploration. In section 5.3 we give evidence that this relationship can be extended to half-K3 surfaces (i.e. blow-ups of $\mathbb{P}^{2}$ at nine points) at certain moduli. In addition to this, the form in which the relevant skew-holomorphic Jacobi forms are found points toward a concrete construction in terms of a vertex algebra attached to a certain indefinite lattice.

The plan of this note is as follows. In section 2 we give a brief review of skewholomorphic Jacobi forms. In section 3 we discuss $S^{1}$ heterotic string compactifications at rational points in Narain moduli space and highlight the connection between the BPS counting function and skew-holomorphic Jacobi forms. In section 4 we review the M5brane elliptic genus, and show that, when evaluated at a relevant attractor point in moduli space, it gives a skew-holomorphic Jacobi form of weight -1 . In section 5 we discuss several examples where weight 2 skew-holomorphic (mock) Jacobi forms that are implicated in moonshine appear. The discussion of $M_{12}$ and del Pezzo surfaces appears in section 5.2, and this is extended to half-K3 surfaces in section 5.3. Some further details and supporting data for these relationships appears in appendix A.

\section{Skew-holomorphic Jacobi forms}

We briefly review skew-holomorphic Jacobi forms in this section, referring to [1] or [15] for more details.

In very general terms, a skew-holomorphic Jacobi form of weight $k$ and index $m$ is a function of the form

$$
\varphi(\tau, z)=\sum_{r \bmod 2 m} \overline{f_{r}(\tau)} \theta_{m, r}(\tau, z)
$$

where the theta-coefficients $f_{r}$ are the components of a holomorphic vector-valued modular form of weight $k-\frac{1}{2}$. In this work we consider $m \in \frac{1}{2} \mathbb{Z}$, and use

$$
\theta_{m, r}(\tau, z):=\sum_{\substack{\ell \in \mathbb{Z}+m \\ \ell=r \bmod 2 m}} \mathrm{e}(m \ell) y^{\ell} q^{\frac{\ell^{2}}{4 m}}
$$


for $r \in \mathbb{Z}+m$, where $\mathrm{e}(x):=e^{2 \pi i x}$ and $y:=\mathrm{e}(z)$ and $q:=\mathrm{e}(\tau)$. Usually it is required that $f_{r}(\tau)=O(1)$ as $\Im(\tau) \rightarrow \infty$, for all $r$, and the term weakly skew-holomorphic is used when this is relaxed to $f_{r}(\tau)=O\left(e^{C \Im(\tau)}\right)$ for some $C>0$. A skew-holomorphic mock Jacobi form is a function as in (2.1) for which the $f_{r}$ are mock modular forms in the usual sense (cf. e.g. [1]).

In order to formulate some examples define the thetanullwerte

$$
\begin{aligned}
\theta_{m, r}^{0}(\tau) & :=\sum_{\substack{\ell \in \mathbb{Z}+m \\
\ell=r \bmod 2 m}} \mathrm{e}(m \ell) q^{\frac{\ell^{2}}{4 m}}, \\
\theta_{m, r}^{1}(\tau):= & \sum_{\substack{\ell \in \mathbb{Z}+m \\
\ell=r \bmod 2 m}} \mathrm{e}(m \ell) \ell q^{\frac{\ell^{2}}{4 m}} .
\end{aligned}
$$

Then for $k \in\{1,2\}$ and $m \in \frac{1}{2} \mathbb{Z}$ the function

$$
t_{k, m}(\tau, z):=\sum_{r \bmod 2 m} \overline{\theta_{m, r}^{k-1}(\tau)} \theta_{m, r}(\tau, z)
$$

is a skew-holomorphic Jacobi form of weight $k$ and index $m$. These theta-type skewholomorphic Jacobi forms (cf. section 3.1 of [15]) arise as shadows in umbral moonshine. For example, if

$$
H^{(2)}(\tau)=-2 q^{-\frac{1}{8}}+90 q^{\frac{7}{8}}+462 q^{\frac{15}{8}}+1540 q^{\frac{23}{8}}+\ldots
$$

is the McKay-Thompson series attached to the identity element of $M_{24}$ by Mathieu moonshine [2] then $\phi^{(2)}(\tau, z):=H^{(2)}(\tau)\left(\theta_{2,-1}(\tau, z)-\theta_{2,1}(\tau, z)\right)$ is a (weakly holomorphic) mock Jacobi form of weight 1 and index 2 , and its shadow is proportional to $t_{2,2}(\tau, z)$.

The half-integral index theta series (2.2), (2.3) include some familiar examples, which will play a role in section 5 . For instance, for $m=\frac{1}{2}$ we have

$$
\begin{aligned}
\theta_{\frac{1}{2}, \frac{1}{2}}(\tau, z) & =i \sum_{n \in \mathbb{Z}} y^{n+\frac{1}{2}} q^{\frac{1}{2}\left(n+\frac{1}{2}\right)^{2}} \\
& =i y^{\frac{1}{2}} q^{\frac{1}{8}} \prod_{n>0}\left(1-y^{-1} q^{n}\right)\left(1-y q^{n}\right)\left(1-q^{n}\right) .
\end{aligned}
$$

So $\theta_{\frac{1}{2}, \frac{1}{2}}^{0}$ vanishes identically, but $\theta_{\frac{1}{2}, \frac{1}{2}}^{1}=i \eta^{3}$, where $\eta$ denotes the Dedekind eta function, $\eta(\tau):=q^{\frac{1}{24}} \prod_{n>0}\left(1-q^{n}\right)$. For $m=\frac{3}{2}$ we have $\theta_{\frac{3}{2}, \frac{3}{2}}^{0}=0$ and

$$
\theta_{\frac{3}{2}, \pm \frac{1}{2}}^{0}(\tau)=\mp i \sum_{n \in \mathbb{Z}}(-1)^{n} q^{\frac{1}{6}\left(3 n \pm \frac{1}{2}\right)^{2}}=\mp i \eta(\tau) .
$$

Also note the identity $t_{2, \frac{1}{2}}(\tau, z)=\frac{1}{2} t_{2,2}\left(\tau, \frac{1}{2} z\right)$, which hints at an index $m=\frac{1}{2}$ formulation of Mathieu moonshine. A broader context for this is given in [16].

From a number theoretic point of view skew-holomorphic Jacobi forms play a complementary role to holomorphic Jacobi forms in a particular formulation of the Shimura correspondence, developed by Skoruppa and Zagier [6, 17, 18]. Consequently there are Waldspurger-type results relating Fourier coefficients of holomorphic and (non theta-type) 
skew-holomorphic Jacobi forms of weight at least 2 to special values of $L$-functions of cuspidal modular forms with level (cf. [18]). This mechanism plays an important role in the arithmetic geometry of elliptic curves according to the celebrated Birch-Swinnerton-Dyer conjecture. Applications to moonshine have appeared, for instance, in [15] and [19].

Our focus in section 5 will be on examples of M5-brane configurations that produce (weakly) skew-holomorphic (mock) Jacobi forms of weight 2.

\section{Rational heterotic string compactifications}

In this section we analyze examples of $S^{1}$ compactifications of the heterotic string at points in the Narain moduli space that correspond to rational conformal field theories. By definition these are points at which there is an extended chiral algebra with the CFT containing a finite number of irreducible representations of the chiral algebra. The partition function thus decomposes into a finite sum of the form

$$
Z(q)=\sum_{j, \bar{j}} N_{j \bar{j}} \chi_{j}(q) \bar{\chi}_{\bar{j}}(\bar{q})
$$

where the $N_{j \bar{j}}$ are non-negative integers and the $\chi_{j}\left(\bar{\chi}_{\bar{j}}\right)$ furnish holomorphic (antiholomorphic) irreducible characters of the extended chiral algebra which is larger than the Virasoro algebra. Of course, the $\chi_{j}$ and $\bar{\chi}_{\bar{j}}$ are in general reducible with respect to the Virasoro algebra and decompose into a possibly infinite sum of its irreducible characters. We show that the half-BPS state counting functions which arise can be written in terms of skew-holomorphic Jacobi forms. See [20] for a general discussion of the relationship between rational CFT and attractor points in the moduli space of string compactifications.

\subsection{The rational Gaussian model}

The $c=1$ Gaussian model, corresponding to string compactification on a $S^{1}$ of radius $R$, is defined by an embedding of the unique unimodular even lattice of signature $(1,1)$ into $\mathbb{R}^{1,1}$. We denote the embedded lattice by $\Gamma^{1,1}$ and write lattice vectors and their standard projections as $p=\left(p_{L}, p_{R}\right)$. More generally, for $r=s \bmod 8$, we will use $\Gamma^{r, s}$ to denote an embedding of the unique unimodular even lattice of signature $(r, s)$ into $\mathbb{R}^{r, s}$. Using conventions in which the inverse string tension is $\alpha^{\prime}=2$ we have

$$
\begin{aligned}
& p_{L}=\frac{n}{R}+\frac{w R}{2} \\
& p_{R}=\frac{n}{R}-\frac{w R}{2}
\end{aligned}
$$

with $n, w \in \mathbb{Z}$. The moduli space of the $c=1$ Gaussian model is

$$
\mathbb{Z}_{2} \backslash O(1,1 ; \mathbb{R}) / O(1) \times O(1) \simeq \mathbb{Z}_{2} \backslash \mathbb{R}_{+}
$$

where the $\mathbb{Z}_{2}$ acts as T-duality, $R \mapsto \frac{2}{R}$. Thus the moduli space is the half line $[\sqrt{2}, \infty)$ parametrized by $R$. 
The model contains holomorphic and anti-holomorphic $\mathrm{U}(1)$ currents $J, \bar{J}$ with eigenvalues proportional to $p_{L}, p_{R}$. Introducing chemical potentials $\zeta=\left(\zeta_{L}, \zeta_{R}\right)$ to keep track of these $\mathrm{U}(1)$ charges leads to the partition function

$$
Z(\tau, \zeta)=\Theta_{1,1}(R ; \tau, \zeta)|\eta(\tau)|^{-2}
$$

where

$$
\Theta_{1,1}(R ; \tau, \zeta):=\sum_{p \in \Gamma^{1,1}} q^{\frac{1}{2} p_{L}^{2}} \bar{q}^{\frac{1}{2} p_{R}^{2}} e^{2 \pi i \zeta \cdot p} .
$$

Let $\Gamma_{R}:=\left\{\left(0, p_{R}\right) \in \Gamma^{1,1}\right\}$ be the lattice of right-moving momenta. We now consider rational points in the moduli space where $R^{2} \in \mathbb{Q}$, and say that $\Gamma_{R}$ is generated by $p_{0}$. In order to facilitate the comparison to skew-holomorphic Jacobi forms using the conventions of the previous section we specialize to the case $\zeta(z)=\bar{z} p_{0}$ (this corresponds to choosing the normalization of $\bar{J}$ such that the associated charge has integer eigenvalues). We will show that the Siegel-Narain theta function $\Theta_{1,1}$ is the complex conjugate of a weight one skew-holomorphic Jacobi form of theta-type at such rational points.

Consider first the self-dual point $R=\sqrt{2}$. We then have

$$
\Theta_{1,1}(\sqrt{2} ; \tau, \zeta(z))=\sum_{n, w \in \mathbb{Z}} q^{\frac{(n+w)^{2}}{4}} \bar{q}^{\frac{(n-w)^{2}}{4}} \bar{y}^{n-w}
$$

with $\bar{y}=\mathrm{e}(-\bar{z})$. Breaking the sum into terms with $n+w$ even and $n+w$ odd gives

$$
\Theta_{1,1}(\sqrt{2} ; \tau, \zeta(z))=\sum_{r \bmod 2} \overline{\theta_{1, r}(\tau, z)} \theta_{1, r}^{0}(\tau)=\overline{t_{1,1}(\tau, z)}
$$

which is of the claimed form.

It is not difficult to generalize this to general rational $\frac{R^{2}}{2}$, a problem which appears as Exercise 10.21 in [21]. We write $R^{2}=2 \frac{\kappa^{\prime}}{\kappa}$ with $\kappa^{\prime}, \kappa$ coprime integers. We then have

$$
\begin{aligned}
\Theta_{1,1}\left(\sqrt{2 \frac{\kappa^{\prime}}{\kappa}} ; \tau, \zeta(z)\right) & =\sum_{\left(p_{L}, p_{R}\right) \in \Gamma^{1,1}} q^{\frac{1}{2} p_{L}^{2}} \bar{q}^{\frac{1}{2} p_{R}^{2}} \bar{y}^{p_{R} \sqrt{2 \frac{\kappa^{\prime}}{\kappa}}} \\
& =\sum_{n, w} q^{\frac{\left(n \kappa+w \kappa^{\prime}\right)^{2}}{4 \kappa \kappa^{\prime}}} \bar{q}^{\frac{\left(n \kappa-w \kappa^{\prime}\right)^{2}}{4 \kappa \kappa^{\prime}}} \bar{y}^{n \kappa-w \kappa^{\prime}} .
\end{aligned}
$$

Now define $r_{0}, s_{0}$ to be integers for which $\kappa r_{0}-\kappa^{\prime} s_{0}=1$, which is always possible since $\kappa, \kappa^{\prime}$ are coprime. Define $\omega_{0}$ and $r$ to be the values of $\kappa r_{0}+\kappa^{\prime} s_{0}$ and $n \kappa+w \kappa^{\prime}$ modulo $2 \kappa \kappa^{\prime}$ respectively. Then a short computation shows that $n \kappa-w \kappa^{\prime}=\omega_{0} r \bmod 2 \kappa \kappa^{\prime}$ which allows us to write

$$
\begin{aligned}
\Theta_{1,1}\left(\sqrt{2 \frac{\kappa^{\prime}}{\kappa}} ; \tau, \zeta(z)\right) & =\sum_{r \bmod 2 \kappa \kappa^{\prime}} \sum_{n=\omega_{0} r \bmod 2 \kappa \kappa^{\prime}} \bar{q}^{\frac{n^{2}}{4 \kappa \kappa^{\prime}}} \bar{y}^{n} \sum_{m=r \bmod 2 \kappa \kappa^{\prime}} q^{\frac{m^{2}}{4 \kappa \kappa^{\prime}}} \\
& =\sum_{r \bmod 2 \kappa \kappa^{\prime}} \overline{\theta_{\kappa \kappa^{\prime}, \omega_{0} r}(\tau, z)} \theta_{\kappa \kappa^{\prime}, r}^{0}(\tau)
\end{aligned}
$$


This is almost of the desired form except for the factor of $\omega_{0}$. This factor can be understood in terms of an automorphism of the fusion rule algebra as discussed in [20,22] and in the mathematical literature is related to well-known objects, namely the Eichler-Zagier operators which played a prominent role in [3].

To see this, we can perform a trivial rewriting of the previous equation,

$$
\Theta_{1,1}\left(\sqrt{2 \frac{\kappa^{\prime}}{\kappa}} ; \tau, \zeta(z)\right)=\sum_{s, r \bmod 2 \kappa \kappa^{\prime}} \overline{\theta_{\kappa \kappa^{\prime}, s}(\tau, z)} \delta_{s, \omega_{0} r} \theta_{\kappa \kappa^{\prime}, r}^{0}(\tau) .
$$

The matrix with matrix elements $\delta_{s, \omega_{0} r}$ is an Eichler-Zagier matrix,

$$
\Omega_{\kappa \kappa^{\prime}}(\kappa)_{s r}=\delta_{s, \omega_{0} r},
$$

see [3] for conventions. Recall that $\Omega_{m}(n)_{s r}=1$ if $s+r=0 \bmod 2 n$ and $s-r=0 \bmod \frac{2 m}{n}$, and 0 otherwise. An easy calculation shows that the two conditions required for a matrix element of $\Omega_{\kappa \kappa^{\prime}}(\kappa)$ to be nonzero are equivalent to $s=\omega_{0} r \bmod 2 \kappa \kappa^{\prime}$ :

$$
\begin{aligned}
\left(s-\omega_{0} r\right) \bmod 2 \kappa & =s-\left(\kappa r_{0}+\kappa^{\prime} s_{0}\right) r \bmod 2 \kappa \\
& =s+\left(\kappa r_{0}-\kappa^{\prime} s_{0}\right) r \bmod 2 \kappa \\
& =(s+r) \bmod 2 \kappa \\
\left(s-\omega_{0} r\right) \bmod 2 \kappa^{\prime} & =s-\left(\kappa r_{0}+\kappa^{\prime} s_{0}\right) r \bmod 2 \kappa^{\prime} \\
& =s-\left(\kappa r_{0}-\kappa^{\prime} s_{0}\right) r \bmod 2 \kappa^{\prime} \\
& =(s-r) \bmod 2 \kappa^{\prime}
\end{aligned}
$$

from which it easily follows that $\Omega_{\kappa \kappa^{\prime}}(\kappa)_{s r}=\delta_{s, \omega_{0} r}$ and thus that (3.11) is the complex conjugate of a skew-holomorphic Jacobi form:

$$
\Theta_{1,1}\left(\sqrt{2 \frac{\kappa^{\prime}}{\kappa}} ; \tau, \zeta(z)\right)=\overline{\theta_{\kappa \kappa^{\prime}}(\tau, z)} \cdot \Omega_{\kappa \kappa^{\prime}}(\kappa) \cdot \theta_{\kappa \kappa^{\prime}}^{0}(\tau)
$$

where we have suppressed the vector indices in the above equation.

\subsection{Heterotic strings with Wilson lines}

We now explain the relevance of this computation to BPS state counting for heterotic strings on $S^{1}$. In this case the Narain moduli space has dimension 17 , corresponding to the radius of the $S^{1}$ and a choice of Wilson lines in the Cartan subalgebra of $E_{8} \times E_{8}$ or $\operatorname{Spin}(32) / \mathbb{Z}_{2}$. Half-BPS states correspond to right-moving ground states with arbitrary left-moving excitations [23] and have squared mass proportional to $p_{R}^{2}$. The generating function for these BPS states, summed over all $p_{R}^{2}$ and weighted by a chemical potential for $p_{R}$ is given by ${ }^{1}$

$$
Z_{\mathrm{BPS}}(\tau, \zeta)=\Theta_{17,1}(\tau, \zeta) \eta^{-24}(\tau)
$$

\footnotetext{
${ }^{1}$ For the purpose of comparing to black hole microstate counts, we comment that the partition function defined here has the same leading asymptotic behavior as the familiar $1 / \eta^{24}(\tau)$, receiving only subleading corrections from the theta function. Similar comments apply to the rest of the counting functions considered in this paper.
} 
where now

$$
\Theta_{17,1}(\tau, \zeta):=\sum_{p \in \Gamma^{17,1}} q^{\frac{1}{2} p_{L}^{2}} \bar{q}^{\frac{1}{2} p_{R}^{2}} e^{2 \pi i \zeta \cdot p} .
$$

We expect that $Z_{\mathrm{BPS}}$ can be written in terms of skew-holomorphic Jacobi forms at rational points in the Narain moduli space

$$
\mathcal{N}_{17,1}:=O(17,1, \mathbb{Z}) \backslash O(17,1, \mathbb{R}) / O(17) \times O(1) .
$$

We will show this explicitly for two examples below, and defer comments about the general case to section 3.3 .

The first example involves considering points in the moduli space (3.18) where the Wilson lines are turned off. At these points, the embedded lattice $\Gamma^{17,1}$ respects the standard splitting $\mathbb{R}^{17,1}=\mathbb{R}^{16} \oplus \mathbb{R}^{1,1}$ in the sense that $L:=\Gamma^{17,1} \cap \mathbb{R}^{16}$ is a positive-definite even unimodular lattice with rank 16 and $\Gamma^{17,1} \cap \mathbb{R}^{1,1}$ is unimodular and even with signature $(1,1)$. If we further specialize to points in the moduli space where the $\Gamma^{1,1}$ corresponds to a rational CFT of radius $R=\sqrt{2 \frac{\kappa^{\prime}}{\kappa}}$ we then find

$$
\begin{aligned}
Z_{\mathrm{BPS}}(\tau, \zeta(z)) & =\Theta_{17,1}(\tau, \zeta(z)) \eta^{-24}(\tau) \\
& =\sum_{\left(p_{L}, p_{R}\right) \in \Gamma^{17,1}} q^{\frac{1}{2} p_{L}^{2}} \bar{q}^{\frac{1}{2} p_{R}^{2}} \bar{y}^{p_{R} \sqrt{2 \kappa^{\prime} \kappa}} \\
& =\overline{\theta_{\kappa \kappa^{\prime}}(\tau, z)} \cdot \Omega_{\kappa \kappa^{\prime}}(\kappa) \cdot \theta_{\kappa \kappa^{\prime}}^{0}(\tau) \Theta_{L}(\tau) \eta^{-24}(\tau)
\end{aligned}
$$

where $\Theta_{L}$ is the theta-function attached to the lattice $L$. There are only two even unimodular lattices of rank 16; namely $E_{8} \oplus E_{8}$ and $D_{16}^{+}$. In both cases $\Theta_{L}$ is the unique weight 8 modular form that satisfies $\Theta_{L}(\tau)=1+O(q)$ as $\Im(\tau) \rightarrow \infty$. The partition function $Z_{\mathrm{BPS}}(\tau, \zeta(z))$ is the complex conjugate of a weakly skew-holomorphic Jacobi form of weight -3 .

We can obtain a more subtle rational point by utilizing a construction due to Nikulin [24]. Let $\Lambda_{L}$ be an even, rank 7, positive-definite lattice which is primitively embedded into an even, unimodular, rank 24 lattice $N$, thus $N$ is the Leech lattice or one of the 23 Niemeier lattices. Let $\Lambda_{R}$ be a negative-definite lattice bijectively isometric to $\Lambda_{L}$ (up to an overall minus sign in the quadratic form) and primitively embedded as a sublattice of the (negative-definite) $E_{8}$ root lattice. Define $\Gamma_{L}:=\Lambda_{L}^{\perp} \cap N$ and $\Gamma_{R}:=\Lambda_{R}^{\perp} \cap\left(-E_{8}\right)$ to be the orthogonal complements of $\Lambda_{L}$ and $\Lambda_{R}$ respectively. Then the lattice

$$
\Gamma:=\bigoplus_{i}\left(\left(\Gamma_{L}, 0\right) \oplus\left(0, \Gamma_{R}\right)+\left(g_{L}^{(i)}, g_{R}^{(i)}\right)\right)
$$

is an even, unimodular lattice of signature $(17,1)$. In the above $g_{L}^{(i)}$ and $g_{R}^{(i)}$ are glue vectors which run over the non-trivial elements of the discriminant group of $\Gamma_{L}, \Gamma_{R}$, such that the $\operatorname{map} g_{R}^{(i)} \rightarrow g_{L}^{(i)}$ is an isometry.

Our second example will use this construction for the choice $\Lambda_{L}=E_{7}$ which is embedded in the Niemeier lattice with root system $A_{17} E_{7}$, and $\Lambda_{R}=\left(-E_{7}\right)$ which is primitively embedded into $\left(-E_{8}\right) .{ }^{2}$ Our conventions for the $A_{17} E_{7}$ root lattice are as follows. Take

\footnotetext{
${ }^{2}$ For details on the construction of such lattices, see e.g. [25, 26].
} 
$e_{1}, e_{2}, \ldots, e_{26}$ to be an orthonormal basis for $\mathbb{R}^{26}=\mathbb{R}^{8} \oplus \mathbb{R}^{18}$ and take the $E_{7}$ root lattice to be embedded in the first $\mathbb{R}^{8}$ with simple roots $r_{i}:=e_{i+2}-e_{i+1}$ for $i=1,2, \ldots, 6$, and $r_{7}:=\frac{1}{2}\left(e_{1}+e_{2}+e_{3}+e_{4}-e_{5}-e_{6}-e_{7}-e_{8}\right)$. For the $A_{17}$ root system we embed in the $\mathbb{R}^{18}$ factor and take the simple roots to be $r_{i}:=e_{i}-e_{i+1}$ for $i=9,10, \ldots, 26$. Recall the construction of the Niemeier lattice $N$ corresponding to the $A_{17} E_{7}$ root system. Letting $L^{\star}$ denote the dual of a lattice $L$, we have embeddings

$$
A_{17} E_{7} \subset N \subset\left(A_{17} E_{7}\right)^{\star}
$$

which implies that $N / A_{17} E_{7}$ is a subgroup of $\left(A_{17} E_{7}\right)^{\star} / A_{17} E_{7}$. Moreover, since $N$ is an even lattice, it is an isotropic subgroup, meaning that the quadratic form of the discriminant group restricted to $N / A_{17} E_{7}$ vanishes. Now, the discriminant group of $A_{17} E_{7}$ is $\mathbb{Z}_{2} \times \mathbb{Z}_{18}$ from the $E_{7}$ and $A_{17}$ factors respectively.

The $\mathbb{Z}_{2}$ component of the discriminant group is generated by

$$
v:=\frac{1}{4}\left(3 e_{1}-e_{2}-e_{3}-e_{4}-e_{5}-e_{6}+3 e_{7}-e_{8}\right)
$$

while the $\mathbb{Z}_{18}$ is generated by

$$
w:=\frac{1}{18}\left(17 e_{9}-\sum_{i=10}^{26} e_{i}\right) .
$$

One can check that the quadratic form on the discriminant group vanishes on the isotropic subgroup $\langle v+3 w\rangle \simeq \mathbb{Z}_{6}$. The Niemeier lattice is obtained as

$$
N=\bigcup_{n=0, \ldots, 5}\left(A_{17} E_{7}+n(v+3 w)\right)
$$

and the orthogonal complement of the $E_{7}$ root lattice in $N$ is easily seen to be

$$
\Gamma_{L}=A_{17} \cup\left(A_{17}+6 w\right) \cup\left(A_{17}+12 w\right) .
$$

One can convince oneself that the discriminant group $\Gamma_{L}^{\star} / \Gamma_{L}=\langle 3 w\rangle \simeq \mathbb{Z}_{2}$.

The even unimodular lattice $\Gamma$ that we obtain in this way satisfies

$$
\Theta_{17,1}(\tau, \zeta(z))=\Theta_{\Gamma_{L}}(\tau) \overline{\theta_{1,0}(\tau, z)}+\Theta_{\Gamma_{L}+3 w}(\tau) \overline{\theta_{1,1}(\tau, z)},
$$

and an explicit computation of the theta coefficients yields

$$
\begin{aligned}
\Theta_{\Gamma_{L}}(\tau)= & 1+306 q+55488 q^{2}+1161984 q^{3}+10054242 q^{4} \\
& +53585088 q^{5}+210351744 q^{6}+668519424 q^{7}+\cdots, \\
\Theta_{\Gamma_{L}+3 w}(\tau)= & 1632 q^{5 / 4}+134912 q^{9 / 4}+2110176 q^{13 / 4} \\
& +15898368 q^{17 / 4}+76968384 q^{21 / 4}+286866432 q^{25 / 4}+\cdots .
\end{aligned}
$$

We can identify the above theta coefficients further using results in [5]. Skoruppa classifies the weight $k$ index 1 skew-holomorphic Jacobi forms:

$$
J_{k, 1}^{\mathrm{sk}}=M_{k-1}\left(\mathrm{SL}_{2}(\mathbb{Z})\right) \cdot t_{1,1}(\tau, z) \oplus M_{k-3}\left(\mathrm{SL}_{2}(\mathbb{Z})\right) \cdot U(\tau, z) .
$$


Here, $M_{k}\left(\mathrm{SL}_{2}(\mathbb{Z})\right)$ is the space of weight $k$ holomorphic modular forms for $\mathrm{SL}_{2}(\mathbb{Z})$, and

$$
\begin{aligned}
U(\tau, z) & :=\frac{12}{\pi i} \frac{\partial}{\partial \bar{\tau}} t_{1,1}(\tau, z)+\overline{E_{2}(\tau)} t_{1,1}(\tau, z), \\
E_{2}(\tau) & :=1-24 \sum_{\ell \geq 1}\left(\sum_{d \mid \ell} d\right) q^{\ell}
\end{aligned}
$$

Letting $E_{k}(\tau)=1+O(q)$ be the Eisenstein series of weight $k$, the weight nine skewholomorphic forms (and in particular the function we found above) should be of the form

$$
a \overline{E_{4}(\tau)^{2}} t_{1,1}(\tau, z)+b \overline{E_{6}(\tau)} U(\tau, z)
$$

One can verify that the theta function we computed earlier corresponds to the choice $a=\frac{5}{6}$ and $b=\frac{1}{6}$,

$$
\Theta_{17,1}(\tau, \zeta(z))=\frac{1}{6}\left(5 \overline{E_{4}(\tau)^{2}} t_{1,1}(\tau, z)+\overline{E_{6}(\tau)} U(\tau, z)\right)
$$

We are then left with the BPS counting function

$$
Z_{\mathrm{BPS}}(\tau, \zeta(z))=\frac{1}{6}\left(5 E_{4}(\tau)^{2} \overline{t_{1,1}(\tau, z)}+E_{6}(\tau) \overline{U(\tau, z)}\right) \eta^{-24}(\tau)
$$

It should not be hard to generalize this analysis to other rational points in the moduli space (3.18) at which the BPS state counting function can be expressed in terms of skewholomorphic Jacobi forms.

\subsection{Rational toroidal compactifications}

As a technical aside, we would like to briefly sketch the general construction which underlies the examples of the previous sections. Quite generally, toroidal string compactifications correspond to Narain lattices $\Gamma$ of signature $(d+8 s, d)$. The points in the moduli space of such lattices where the associated CFT becomes rational are specified by triples $\left(\Gamma_{L}, \Gamma_{R}, \phi\right)$, where we demand that $\phi: \Gamma_{R}^{\star} / \Gamma_{R} \rightarrow \Gamma_{L}^{\star} / \Gamma_{L}$ be an isometric bijection of the discriminant groups. The discriminant groups $\Gamma_{L}^{\star} / \Gamma_{L}$ and $\Gamma_{R}^{\star} / \Gamma_{R}$ inherit their norms from the norms on $\Gamma_{L}^{\star}$ and $\Gamma_{R}^{\star}$ reduced modulo 2 . Using $\phi$ to obtain so-called glue vectors $(\phi(\lambda), \lambda)$, we may construct the full, rational, unimodular lattice from this data as

$$
\Gamma:=\bigcup_{\lambda \in \Gamma_{R}^{\star} / \Gamma_{R}}\left(\Gamma_{L} \oplus \Gamma_{R}+(\phi(\lambda), \lambda)\right) .
$$

It easily follows that the Siegel-Narain theta function admits the decomposition

$$
\Theta_{\Gamma}(\tau)=\sum_{\lambda \in \Gamma_{R}^{\star} / \Gamma_{R}} \Theta_{\Gamma_{L}+\phi(\lambda)}(\tau) \overline{\Theta_{\Gamma_{R}+\lambda}(\tau)}
$$

where we have defined

$$
\Theta_{L+\lambda}(\tau):=\sum_{\gamma \in L+\lambda} q^{\frac{\gamma^{2}}{2}}
$$


for an arbitrary positive-definite, even lattice $L$. In this construction, $\Gamma_{L}:=\left\{\left(p_{L}, 0\right) \in \Gamma\right\}$ is the lattice of purely left-moving momenta, and similarly for $\Gamma_{R}$. See section 10.2 of [20] for a more detailed discussion.

In the previous sections, we specialized to $d=1$ and exploited the fact that the rightmoving momentum lattice must be of the form $\Gamma_{R} \simeq \sqrt{2 m} \mathbb{Z}$ with associated theta-function

$$
\Theta_{\sqrt{2 m} \mathbb{Z}+r}(\tau)=\theta_{m, r}^{0}(\tau)
$$

for $r$ in $\Gamma_{R}^{\star} / \Gamma_{R} \simeq \mathbb{Z}_{2 m}$. Indeed, upon flavoring by an additional $\bar{J}$ quantum number, we find that the points in the moduli space with $\Gamma_{R} \simeq \sqrt{2 m} \mathbb{Z}$ recovered (complex conjugates of) index $m$ skew-holomorphic Jacobi forms.

In this language, the $c=1$ Gaussian model with radius $R=\sqrt{2 \frac{\kappa^{\prime}}{\kappa}}$ corresponds to the triple $\left(\sqrt{2 \kappa^{\prime} \kappa} \mathbb{Z}, \sqrt{2 \kappa^{\prime} \kappa} \mathbb{Z}, r \rightarrow \omega_{0} r\right)$, with the gluing of left and right-moving momentum lattices specified by the isometry "multiplication by $\omega_{0} . "$ The different choices of isometries give rise to different Eichler-Zagier matrices $\Omega_{\kappa \kappa^{\prime}}(\kappa)$ which commute with the action of the modular group on the thetanullwerte. Similar comments should apply to the problem of classifying the rational points in the moduli space of the heterotic string with Wilson lines, as well as the skew-holomorphic Jacobi forms which arise.

We now turn to a richer source of strings - those arising from wrapped M5-branes - and show that their associated elliptic genera can also be expressed in terms of skewholomorphic Jacobi forms.

\section{The M5-brane elliptic genus}

Here we review basic facts about the worldsheet theory on a wrapped M5-brane.

\subsection{Multiplets}

The M5-brane wrapping a divisor in a Calabi-Yau threefold gives rise, at low-energies, to an effective string, sometimes called an "MSW string," with $(0,4)$ worldsheet supersymmetry. This theory was studied in detail from various viewpoints in, e.g., [9-11, 27]. Suppose the M5-brane is wrapping a divisor $\mathcal{P}$ in a Calabi-Yau threefold $X$. Then the low-energy theory on the effective string (arising from dimensional reduction of the M5-brane worldvolume fields) is as follows.

Consider the inclusion map

$$
i: \mathcal{P} \rightarrow X .
$$

This naturally gives rise to a pullback map $i^{*}: H^{2}(X, \mathbb{Z}) \rightarrow H^{2}(\mathcal{P}, \mathbb{Z})$. We define $\Lambda$ to be $i^{*}\left(H^{2}(X, \mathbb{Z})\right)$ equipped with the bilinear form given by $(A \mid B):=-\int_{\mathcal{P}} A \wedge B$. The pullback two-forms $i^{*} \alpha \in H^{2}(\mathcal{P}, \mathbb{Z})$ can be associated with chiral worldvolume fields in the $(0,4)$ worldsheet $\sigma$-model as follows.

- Self-dual two-forms on $\mathcal{P}$ that extend non-trivially to $X$ give rise to left-moving scalars on the worldsheet.

- Anti self-dual two-forms on $\mathcal{P}$ that extend non-trivially to $X$ give rise to right-moving scalars on the worldsheet. 
In fact, for a Calabi-Yau threefold $X$, the Kähler form is the only two-form that pulls back to an anti self-dual form on $\mathcal{P}$. As a result there are $b^{2}(X)-1$ left-moving scalars and 1 right-moving scalar coming from these sources.

It is important to remember that the worldsheet fields include universal worldsheet multiplets arising from (super) Goldstone modes. This gives three additional non-chiral scalars that can translate the effective string. The total of four right-moving bosons (including the one arising from the pullback of the Kähler form) have four Fermi superpartners arising from the $(0,4)$ supersymmetry. Zero modes of these fermions lead to a modification of the definition of the M5-brane ellliptic genus relative to the conventional elliptic genus (see (4.1)) as the conventional quantity would vanish in this circumstance.

In a model-dependent way, there are also additional fields present in the generic wrapped M5-brane theory. These parametrize the moduli space of motions of the wrapped divisor in the Calabi-Yau space $X$. Although our subsequent discussion will be independent of these fields it should be mentioned that in the limit of large central charge, where the effective string can sometimes be related to a weakly curved black string, they constitute the most numerous degrees of freedom.

\subsection{The index}

For a fixed M5 theory the worldsheet elliptic genus can be defined as follows. First define

$$
Z^{\prime}(\tau, \zeta):=\operatorname{tr}_{R}\left(F^{2}(-1)^{F} e^{\pi i p \cdot Q} e^{2 \pi i \tau\left(L_{0}-\frac{c_{L}}{24}\right)} e^{-2 \pi i \bar{\tau}\left(\bar{L}_{0}-\frac{c_{R}}{24}\right)} e^{2 \pi i \zeta \cdot Q}\right) .
$$

Here the $\zeta^{a}$ are chemical potentials and the $Q_{a}$ are charges under the $b_{2}(X)$ abelian currents associated with the chiral bosons; i.e. $\left(b_{2}-1\right)$ left-moving currents, and a single right-moving current. The $p^{a}$ parametrize the (discrete) choice of divisor in $H_{4}(X, \mathbb{Z})$ that the M5-brane wraps. The fermion number is defined in the usual way as twice the charge of the U(1) generator in the $\mathrm{SU}(2)_{R}$ R-symmetry which exists in the $\mathcal{N}=4$ superconformal algebra. The extra factor of $F^{2}$ as compared to the conventional elliptic genus is present in order to absorb the fermion zero modes mentioned above.

This quantity isn't quite the one we want to work with, as it includes information about the momenta in the $\mathbb{R}^{3}$ transverse to the effective string in the non-compact directions of space. Instead, the generalized elliptic genus $Z(\tau, \zeta)$ is defined by requiring that

$$
Z^{\prime}(\tau, \zeta)=Z(\tau, \zeta) \int d^{3} \vec{\pi}\left(e^{2 \pi i \tau} e^{-2 \pi i \bar{\tau}}\right)^{\frac{1}{2} \vec{\pi}^{2}}=Z(\tau, \zeta)(2 \operatorname{Im} \tau)^{\frac{3}{2}}
$$

It is easy to see $[10-12]$ that $Z$ has weight $\left(-\frac{3}{2}, \frac{1}{2}\right)$, in the sense that

$$
Z\left(\frac{a \tau+b}{c \tau+d}, \frac{\zeta}{c \tau+d}\right) \frac{(c \tau+d)^{2}}{|c \tau+d|} \mathrm{e}\left(m \frac{c z^{2}}{c \bar{\tau}+d}\right)=\chi\left(\begin{array}{ll}
a & b \\
c & d
\end{array}\right) Z(\tau, \zeta)
$$

for some $m$, and some multiplier $\chi: S L_{2}(\mathbb{Z}) \rightarrow \mathbb{C}$, when $\left(\begin{array}{ll}a & b \\ c & d\end{array}\right) \in S L_{2}(\mathbb{Z})$. This is what we would expect from (the complex conjugate of) a skew-holomorphic Jacobi form of weight -1 (with a multiplier system). The shift in charges under a large gauge transformation 
states that the generalized index admits a decomposition

$$
Z(\tau, \zeta)=\sum_{\mu \in \Lambda^{*} / \Lambda} \Theta_{\mu}(\tau, \zeta) h_{\mu}(\tau)
$$

into Siegel-Narain theta functions

$$
\Theta_{\mu}(\tau, \zeta):=\sum_{Q \in \mu+\Lambda+\frac{p}{2}} \mathrm{e}\left(\frac{\tau}{2}\left(Q_{+} \mid Q_{+}\right)+\frac{\bar{\tau}}{2}\left(Q_{-} \mid Q_{-}\right)+\left(Q \mid \zeta+\frac{p}{2}\right)\right),
$$

where the splitting $Q=Q_{+}+Q_{-}$depends on the Grassmannian $\frac{O\left(b_{2}-1,1\right)}{O\left(b_{2}-1\right) \times O(1)}$. In the sequel we will always set the chemical potentials conjugate to the left-moving currents to zero, and only keep track of the right-moving chemical potential.

Next we will show that, at certain points in the moduli space, the generalized elliptic genus of an MSW string is naturally a skew-holomorphic (mock) Jacobi form. First, recall that in the presence of an MSW string the Calabi-Yau moduli which are vector multiplets in the low-energy supergravity undergo an "attractor flow." That is, they flow to certain specific values at the horizon of the related black string, independent of their values at infinity in $\mathbb{R}^{5}$. This 'attractor mechanism' gives a natural preferred choice of moduli. In M-theory on $X$, the vector multiplet moduli are the Kähler moduli of $X$ (excepting the overall volume, which transforms in a hypermultiplet). At the attractor point in moduli space, the Kähler form $J$ on $X$ satisfies $J \sim p$. As a result one can find the right-moving chiral $\mathrm{U}(1)$ current and its associated charge to be

$$
Q_{-}=\frac{p \cdot Q}{p^{2}} p
$$

As we already know that $Z(\tau, \zeta)$ transforms as a weight $\left(-\frac{3}{2}, \frac{1}{2}\right)$ modular form, what remains to check is that $\overline{Z(\tau, \zeta)}$, for a specific choice of $\zeta=\zeta(z)$, satisfies the elliptic transformation

$$
\overline{Z(\tau, \zeta(z+\lambda \tau+\mu))} \text { e }\left(m\left(\lambda^{2} \tau+2 \lambda z+\lambda+\mu\right)\right)=\overline{Z(\tau, \zeta(z))}
$$

for $\lambda, \mu \in \mathbb{Z}$. This will imply that $\bar{Z}$ admits a decomposition as in (2.1).

Let $\zeta=\bar{z} p$. At the attractor moduli the Siegel-Narain theta function $\Theta_{\mu}(\tau, \bar{\tau}, \zeta)$ becomes

$$
\tilde{\theta}_{\mu}(\tau, \bar{z}):=\sum_{Q \in \mu+\Lambda+\frac{p}{2}}(-1)^{p \cdot Q} q^{\frac{1}{2} Q_{+}^{2}} \bar{q}^{\frac{1}{2} \frac{(p \cdot Q)^{2}}{p^{2}}} e^{2 \pi i \bar{z} p \cdot Q}
$$

where $q=\mathrm{e}(\tau)$ and $\bar{q}=\mathrm{e}(-\bar{\tau})$, and we used (4.6) in writing the power of $\bar{q}$. We can show that

$$
\tilde{\theta}_{\mu}(\tau, \bar{z}+n \bar{\tau}+m)=(-1)^{p^{2}(m+n)} \mathrm{e}\left(\frac{1}{2} p^{2} n^{2}\right) \tilde{\theta}_{\mu}(\tau, \bar{z})
$$

by a shift $Q \mapsto Q+p n$ in the sum. This verifies that, at the attractor point in moduli space, $\overline{Z(\tau, p \bar{z})}$ is a skew-holomorphic Jacobi form of index $\frac{1}{2} p^{2}$ with elliptic variable $z$.

An interesting question for future work would be to determine if there are other (non-attractor) moduli where the M5-brane elliptic genus reduces to a skew-holomorphic Jacobi form. 


\section{$5 \quad$ Examples}

We now discuss several examples of M5-brane elliptic genera computed in [14]. In each case we find a natural relation to a weakly skew-holomorphic Jacobi form of weight 2 that plays a role in a moonshine.

\subsection{The projective plane}

The elliptic genus for one M5-brane wrapping $\mathbb{P}^{2}$ can be written as

$$
Z_{\mathbb{P}^{2}}^{(1)}(\tau, z)=(-i) \theta_{\frac{1}{2}, \frac{1}{2}}(-\bar{\tau},-z) \eta^{-3}(\tau)
$$

(cf. (2.6)) thanks to work of Göttsche [28]. In comparison with section 4 we have kept only the chemical potential for the right-moving $\mathrm{U}(1)$ charge, which we henceforth denote by $z$.

So the function $\overline{Z_{\mathbb{P}^{2}}^{(1)}(\tau, z)}$ is a skew-holomorphic Jacobi form of weight -1 and index $\frac{1}{2}$, and since $\theta_{\frac{1}{2}, \frac{1}{2}}^{1}=i \eta^{3}$ (cf. (2.6)) we may write

$$
Z_{\mathbb{P}^{2}}^{(1)}(\tau, z)=\overline{\varphi_{\mathbb{P}^{2}}^{(1)}(\tau, \bar{z})} \eta^{-6}(\tau)
$$

where $\varphi_{\mathbb{P}^{2}}^{(1)}(\tau, z)=t_{2, \frac{1}{2}}(\tau, z)=\frac{1}{2} t_{2,2}\left(\tau, \frac{1}{2} z\right)$, and $t_{2,2}$ is the weight 2 , index 2 skew-holomorphic Jacobi form that appears as a shadow in Mathieu moonshine (cf. (2.4)).

The connection to Mathieu groups becomes stronger when we consider two M5-branes wrapping $\mathbb{P}^{2}$. To explain this let $H(n)$ denote the Hurwitz class number of binary quadratic forms of discriminant $-n$ when $n>0$, and set $H(0):=-\frac{1}{12}$. Then $\mathscr{H}(\tau):=\sum_{n \geq 0} H(n) q^{n}$ is a mock modular form of weight $\frac{3}{2}$ for $\Gamma_{0}(4)$ with shadow (proportional to) $\theta_{1,0}^{0}$ (cf. (2.3)). This was first discovered by Zagier [29]. Very recent work [30] proves that

$$
24 \mathscr{H}(\tau)=-2+8 q^{3}+12 q^{4}+24 q^{7}+24 q^{8}+\ldots
$$

is the graded dimension of a graded virtual module for the sporadic Mathieu group $M_{11}$, and $48 \mathscr{H}(\tau)=-4+16 q^{3}+24 q^{4}+\ldots$ is the graded dimension of a graded virtual module for $M_{23}$.

Now set $\hat{f}_{j}(\tau):=3 \hat{h}_{j}(\tau) \eta^{-6}(\tau)$ for $j \in\{0,1\}$, where $\hat{h}_{j}$ is the completion of the mock modular form

$$
h_{j}(\tau):=\sum_{n=0}^{\infty} H(4 n+3 j) q^{n+\frac{3 j}{4}} .
$$

Then the elliptic genus of two M5-branes wrapping $\mathbb{P}^{2}$ is given [31-33] by

$$
Z_{\mathbb{P}^{2}}^{(2)}(\tau, z)=\hat{f}_{0}(\tau) \theta_{1,1}(-\bar{\tau},-z)-\hat{f}_{1}(\tau) \theta_{1,0}(-\bar{\tau},-z) .
$$

Similar to (5.2) we may write

$$
Z_{\mathbb{P}^{2}}^{(2)}(\tau, z)=\overline{\varphi_{\mathbb{P}^{2}}^{(2)}(\tau, \bar{z})} \eta^{-6}(\tau)
$$

where $\varphi_{\mathbb{P}^{2}}^{(2)}(\tau, z):=3 \overline{\hat{h}_{0}(\tau)} \theta_{1,0}(\tau, z)-3 \overline{\hat{h}_{1}(\tau)} \theta_{1,1}(\tau, z)$ is a skew-holomorphic mock Jacobi form of weight 2 and index 1 that exhibits moonshine for the Mathieu groups $M_{11}$ and $M_{23}$ 
according to [30]. Thus M5-branes on $\mathbb{P}^{2}$ give a starting point from which we may pursue a geometric understanding of Mathieu moonshine for (rescaled) Hurwitz class numbers.

It is interesting to note that the generating function $\mathscr{H}(\tau)$ also arises as an example of a function counting BPS jumping loci of maximal rank for $K 3 \times T^{2}$, or equivalently, counting attractor black holes, in the precise sense described in [35]. Also, the theta-coefficients of $\varphi$ in (5.5) recur in the elliptic genus for two M5-branes wrapping the Hirzebruch surface $\mathbb{F}_{1}$ (see section 4.2 of [14]). In both these settings, and of course for two M5-branes wrapping $\mathbb{P}^{2}$, it would be interesting to compare geometric twinings with the functions coming from the analysis of [30].

\subsection{Degree one del Pezzo surfaces}

Next we consider M5-branes wrapping a del Pezzo surface of degree 1 (i.e. $\mathbb{P}^{2}$ blown up at 8 points). The elliptic genus was first described in [10]. Start with the Fermat quintic $\left\{\sum_{i} x_{i}^{5}=0\right\} \subset \mathbb{P}^{4}$ and quotient by the $\mathbb{Z}_{5}$ action $x_{i} \rightarrow \omega^{i} x_{i}$ where $\omega:=\mathrm{e}\left(\frac{1}{5}\right)$. The hyperplane section $\mathcal{P}$ of the resulting orbifold has $\chi(\mathcal{P})=11$ and is rigid with $b_{2}^{+}=1$. It has $H^{2}(\mathcal{P}, \mathbb{Z})=\mathbb{Z} \oplus\left(-E_{8}\right)$ and is thus a del Pezzo surface of degree 1 .

For a single M5-brane wrapping $\mathcal{P}$ we have $Z_{\mathrm{dP}_{8}}^{(1)}(\tau, z)=\overline{\varphi_{\mathrm{dP}_{8}}^{(1)}(\tau, \bar{z})} \eta^{-6}(\tau)$ for the elliptic genus, where

$$
\begin{aligned}
\varphi_{\mathrm{dP}_{8}}^{(1)}(\tau, z): & =\overline{E_{4}(\tau) \eta^{-8}(\tau)} t_{2, \frac{1}{2}}(\tau, z) \\
& =\overline{E_{4}(\tau) \eta^{-5}(\tau)}(-i) \theta_{\frac{1}{2}, \frac{1}{2}}(\tau, z) .
\end{aligned}
$$

This is a weakly skew-holomorphic Jacobi form of weight 2 with a multiplier, and may be compared to (5.2).

Inspired by the discussion in section 5.1 we consider the possibility that the coefficients of the anti-holomorphic factor in (5.6) also admit interpretations in terms of representations of Mathieu groups. Observe that

$$
f_{\mathrm{dP}_{8}}^{(1)}(\tau):=E_{4}(\tau) \eta^{-5}(\tau)
$$

is the unique modular form of weight $\frac{3}{2}$ for $S L_{2}(\mathbb{Z})$ that has the same multiplier as $\eta^{-5}$ and satisfies $f_{\mathrm{dP}_{8}}^{(1)}(\tau)=q^{-\frac{5}{24}}+O\left(q^{\frac{19}{24}}\right)$ as $\Im(\tau) \rightarrow \infty$. By considering analogous functions for the congruence subgroups $\Gamma_{0}(n)<S L_{2}(\mathbb{Z})$ we are led to a family $f_{\mathrm{dP}_{8}, n Z}^{(1)}$ of modular forms of weight $\frac{3}{2}$ with various levels which achieves this goal for the sporadic simple Mathieu group $M_{12}$. That is, the $f_{\mathrm{dP}_{8}, n Z}^{(1)}$ serve as trace functions

$$
f_{\mathrm{dP}_{8},[g]}^{(1)}(\tau)=\sum_{d \in \mathbb{Z}+\frac{19}{24}} \operatorname{tr}\left(g \mid W_{\mathrm{dP}_{8}, d}^{(1)}\right) q^{d}
$$

for a graded virtual $M_{12}$-module $W_{\mathrm{dP}_{8}}^{(1)}=\bigoplus_{d} W_{\mathrm{dP}_{8}, d}^{(1)}$ with graded dimension given by (5.7).

Details on the modular forms $f_{\mathrm{dP}_{8}, n Z}^{(1)}$ are given in appendix A, including the first few coefficients in their Fourier expansions (see tables 2-3) and the decompositions of the corresponding $W_{\mathrm{dP}_{8}, d}^{(1)}$ into irreducible modules for $M_{12}$ (see tables 4-8). From that 
information alone it is not immediate that the virtual $M_{12}$-module $W_{\mathrm{dP}_{8}}^{(1)}$ satisfying (5.8) exists, but we can verify this using arguments very similar to those appearing in recent literature on moonshine in weight $\frac{3}{2}$, including $[19,30]$. So we refrain from reproducing the details here.

The reader will note that $f_{\mathrm{dP}_{8}}^{(1)}$ is $\eta^{3}$ times the graded dimension of the basic representation $V_{E_{8}}$ of the affine Lie algebra of type $E_{8}$. This space naturally admits an action by the adjoint Lie group $E_{8}(\mathbb{C})$, so it is natural to ask if the twining functions $f_{\mathrm{dP}_{8},[g]}^{(1)}$ are related to this action. Here we note that $M_{12}$ is not a subgroup of $E_{8}(\mathbb{C})$ according to [34], so the virtual $M_{12}$-module $W_{\mathrm{dP}_{8}}^{(1)}$ cannot be recovered in a simple way from $V_{E_{8}}$.

We obtain an assignment of weakly skew-holomorphic Jacobi forms of weight 2 and index $\frac{1}{2}$ to elements of $M_{12}$ simply by setting

$$
\varphi_{\mathrm{dP}_{8}, n Z}^{(1)}(\tau, z):=(-i) \overline{f_{\mathrm{dP}_{8}, n Z}^{(1)}(\tau)} \theta_{\frac{1}{2}, \frac{1}{2}}(\tau, z) .
$$

These forms in turn define twinings

$$
Z_{\mathrm{dP}_{8}, n Z}^{(1)}(\tau, z):=\overline{\varphi_{\mathrm{dP}_{8}, n Z}^{(1)}(\tau, \bar{z})} \eta^{-6}(\tau)
$$

of the M5-brane elliptic genus $Z_{\mathrm{dP}_{8}}^{(1)}$. As a result, it is natural to ask how the twining functions (5.10) are related to the symmetries of M5-brane theory on $\mathcal{P}$, and whether this relationship between $M_{12}$ and the del Pezzo surface of degree 1 is connected in some way to the original Mathieu moonshine [2], which relates $M_{24}$ to the K3 elliptic genus. It would be interesting to gain a physical or geometric understanding of the twining functions $Z_{\mathrm{dP}_{8}, n Z}^{(1)}$.

For a pair of M5-branes on $\mathcal{P}$ we have $Z_{\mathrm{dP}_{8}}^{(2)}(\tau, z)=\overline{\varphi_{\mathrm{dP}_{8}}^{(2)}(\tau, \bar{z})} \eta^{-6}(\tau)$ where

$$
\varphi_{\mathrm{dP}_{8}}^{(2)}(\tau, z):=\overline{E_{4}^{2}(\tau) \eta^{-16}(\tau)}\left(\overline{\hat{h}_{0}(\tau)} \theta_{1,0}(\tau, z)-\overline{\hat{h}_{1}(\tau)} \theta_{1,1}(\tau, z)\right)
$$

(cf. (5.4)). In light of the discussions above and in section 5.1 it seems likely that naturally defined Mathieu group twinings of $Z_{\mathrm{dP}_{8}}^{(2)}$ also exist. Are there naturally defined twinings of $Z_{\mathrm{dP}_{8}}^{(n)}$ by $g \in M_{12}$ for all $n$ ? What do they tell us about M5-branes on $\mathcal{P}$ ?

\subsection{Half-K3 surfaces}

In this final section we consider the elliptic genus for a single M5-brane wrapping a half-K3 surface (i.e. $\mathbb{P}^{2}$ blown up at 9 points). Such surfaces play an important role in the study of E-strings via geometric engineering.

To compute the genus in question we first discuss the cohomology group $H^{2}\left(\frac{1}{2} \mathrm{~K} 3, \mathbb{Z}\right)$. Geometrically it is generated by the hyperplane class corresponding to the hyperplane intersection with $\mathbb{P}^{2}$, denoted by $H$, and the nine blow-ups $c_{i}$, for $i=1, \ldots, 9$. The quadratic form inherited from the intersection form is then given by $\operatorname{diag}(1,-1, \ldots,-1)$. In fact, this lattice is isomorphic to $\mathbb{Z} \oplus(-\mathbb{Z}) \oplus\left(-E_{8}\right)$, and in particular is unimodular 
(but not even). The corresponding basis is given [36] by

$$
\begin{aligned}
& b_{1}:=3 H+\sum_{i=1}^{8} c_{i}, \quad b_{2}:=-c_{9}, \\
& e_{8}:=H+\sum_{i=6}^{8} c_{i}, \quad e_{i}:=c_{i}-c_{i+1} \text { for } i=1, \ldots 7 .
\end{aligned}
$$

Since the lattice is unimodular there is only one term in the decomposition (4.4) of the genus into theta functions. For the case at hand, the class $\mathcal{P}$ of the surface wrapped by the $M 5$ brane is given by the anti-canonical class

$$
\mathcal{P}=K_{\frac{1}{2} \mathrm{~K} 3}=b_{1}-b_{2} .
$$

The theta function we are interested in will depend on the moduli of the half-K3, and one such modulus is given by the size of the elliptic fiber, denoted here by $\frac{1}{R}$. Taking the shift by $\frac{1}{2} \mathcal{P}$ into account, the Siegel-Narain theta function $(4.5)$ is $\Theta(R ; \tau, z)=$ $E_{4}(\tau) \Theta_{1,1}^{\text {odd }}(R ; \tau, z)$, where

$$
\begin{aligned}
& \Theta_{1,1}^{\text {odd }}(R ; \tau, z):= \\
& \sum_{a, b \in \mathbb{Z}}(-1)^{a+b} q^{\frac{1}{2 R^{2}}\left(R^{2} \frac{(a-b)}{2}+\frac{a+b+1}{2}\right)^{2}} \bar{q}^{\frac{1}{2 R^{2}}\left(R^{2} \frac{(b-a)}{2}+\frac{a+b+1}{2}\right)^{2}} \bar{y}^{\left(R^{2} \frac{(b-a)}{2}+\frac{a+b+1}{2}\right) .}
\end{aligned}
$$

The elliptic genus is given by

$$
Z_{\frac{1}{2} \mathrm{~K} 3}^{(1)}(R ; \tau, z)=E_{4}(\tau) \Theta_{1,1}^{\text {odd }}(R ; \tau, z) \eta^{-12}(\tau) .
$$

The question of moonshine-type phenomena is potentially richer in this setting due to the dependence on the parameter $R$. In this work we refrain from a full analysis and restrict ourselves to some special cases. In preparation for this note that (5.14) specializes to theta-type skew-holomorphic Jacobi forms of half-integral index (cf. (2.4)) at special values of $R$. Indeed, by a similar analysis to that given for $\Theta_{1,1}$ in section 3.1 we obtain

$$
\Theta_{1,1}^{\text {odd }}(\sqrt{2 m} ; \tau, z)=\overline{t_{1, m}(\tau, z)}
$$

when $m \in \mathbb{Z}+\frac{1}{2}$ and $m>0$.

Motivated by the discussions in section 5.1 and section 5.2 we now consider the decomposition $Z_{\frac{1}{2} \mathrm{~K} 3}^{(1)}(\sqrt{2 m} ; \tau)=\overline{\varphi_{\frac{1}{2} \mathrm{~K} 3, m}^{(1)}(\tau, z)} \eta^{-6}(\tau)$, where by $(5.16)$ we have

$$
\varphi_{\frac{1}{2} \mathrm{~K} 3, m}^{(1)}(\tau, z)=\overline{E_{4}(\tau) \eta^{-6}(\tau)} t_{1, m}(\tau, z),
$$

which is a skew-holomorphic Jacobi form of weight 2 and index $m$.

The first case to consider is $m=\frac{1}{2}$, but $t_{1, \frac{1}{2}}$ vanishes identically (cf. (2.6)), so we set this case aside for the moment. The next case is $m=\frac{3}{2}$, where, after applying (2.7) we find that

$$
\varphi_{\frac{1}{2} \mathrm{~K} 3, \frac{3}{2}}^{(1)}(\tau, z)=\overline{E_{4}(\tau) \eta^{-5}(\tau)}(i)\left(\theta_{\frac{3}{2}, \frac{1}{2}}(\tau, z)-\theta_{\frac{3}{2},-\frac{1}{2}}(\tau, z)\right) .
$$


Observe that the anti-holomorphic factor in (5.18) is precisely the same as that which appears in $\varphi_{\mathrm{dP}_{8}}^{(1)}$ (cf. (5.6)), in connection with del Pezzo surfaces of degree 1. So from the discussion in section 5.2 we naturally obtain twinings

$$
\varphi_{\frac{1}{2} \mathrm{~K} 3, \frac{3}{2},[g]}^{(1)}(\tau, z):=\overline{f_{\mathrm{dP}_{8},[g]}^{(1)}(\tau)}(i)\left(\theta_{\frac{3}{2}, \frac{1}{2}}(\tau, z)-\theta_{\frac{3}{2},-\frac{1}{2}}(\tau, z)\right)
$$

(cf. (5.8)) of the weight 2 skew-holomorphic Jacobi form (5.18) by $g \in M_{12}$. This in turn leads to twinings

$$
Z_{\frac{1}{2} \mathrm{~K} 3,[g]}^{(1)}(\sqrt{3} ; \tau, z):=\overline{\varphi_{\frac{1}{2} \mathrm{~K} 3, \frac{3}{2},[g]}^{(1)}(\tau, z)} \eta^{-6}(\tau)
$$

by $g \in M_{12}$ of the single M5-brane elliptic genus for half-K3 surfaces at the modulus $R=\sqrt{3}$.

The vanishing of (5.17) at $m=\frac{1}{2}$ suggests that we modify the elliptic genus by introducing a fermion number operator. This amounts to replacing $t_{1, m}$ with $t_{2, m}$ in (5.17). Indeed, if we define

$$
\widetilde{Z}_{\frac{1}{2} \mathrm{~K} 3}^{(1)}(R ; \tau, z):=E_{4}(\tau) \widetilde{\Theta}_{1,1}^{\text {odd }}(R ; \tau, z) \eta^{-12}(\tau)
$$

where

$$
\begin{aligned}
\widetilde{\Theta}_{1,1}^{\text {odd }}(R ; \tau, z):=\sum_{a, b \in \mathbb{Z}}(-1)^{a+b} & \left(R^{2} \frac{(a-b)}{2}+\frac{a+b+1}{2}\right) q^{\frac{1}{2 R^{2}}\left(R^{2} \frac{(a-b)}{2}+\frac{a+b+1}{2}\right)^{2}} \\
\times & \bar{q}^{\frac{1}{2 R^{2}}\left(R^{2} \frac{(b-a)}{2}+\frac{a+b+1}{2}\right)^{2}} \bar{y}^{\left(R^{2} \frac{(b-a)}{2}+\frac{a+b+1}{2}\right)}
\end{aligned}
$$

then $\widetilde{\Theta}_{1,1}^{\text {odd }}(\sqrt{2 m} ; \tau, z)=\overline{t_{2, m}(\tau, z)}$ when $m \in \mathbb{Z}+\frac{1}{2}$ and $m>0$. In this setting we consider the decomposition $\widetilde{Z}_{\frac{1}{2} \mathrm{~K} 3}^{(1)}(\sqrt{2 m} ; \tau)=\overline{\widetilde{\varphi}_{\frac{1}{2} \mathrm{~K} 3, m}^{(1)}(\tau, z)} \eta^{-4}(\tau)$ so that

$$
\widetilde{\varphi}_{\frac{1}{2} \mathrm{~K} 3, m}^{(1)}(\tau, z)=\overline{E_{4}(\tau) \eta^{-8}(\tau)} t_{2, m}(\tau, z)
$$

is the associated weakly skew-holomorphic Jacobi form of weight 2 and index $m$.

Now comparing with (5.6) we find that (5.23) at $m=\frac{1}{2}$ is precisely the skew-holomorphic Jacobi form of weight 2 and index $\frac{1}{2}$ that appeared in section 5.2 in connection with del Pezzo surfaces of degree 1 . So it is natural to define

$$
\widetilde{\varphi}_{\frac{1}{2} \mathrm{~K} 3, \frac{1}{2},[g]}^{(1)}(\tau, z):=\varphi_{\mathrm{dP}_{8},[g]}^{(1)}(\tau, z)
$$

for $g \in M_{12}$. We then obtain twinings

$$
\widetilde{Z}_{\frac{1}{2} \mathrm{~K} 3,[g]}^{(1)}(1 ; \tau):=\overline{\widetilde{\varphi}_{\frac{1}{2} \mathrm{~K} 3, \frac{1}{2},[g]}^{(1)}(\tau, z)} \eta^{-4}(\tau)
$$

of the modified single M5-brane elliptic genus for half-K3 surfaces by $g \in M_{12}$ when $R=1$. 
We conclude this section with four remarks. Firstly, it is natural to ask if twinings by $M_{12}$ of the elliptic genera (5.15) and (5.21) for half-K3 surfaces can be defined for all $R$. Are there special values of $R$ for which this hidden symmetry extends beyond $M_{12}$ ? Secondly, it would be interesting to compare the physical twinings of the elliptic genera (5.15) and (5.21) with the series (5.20) and (5.25) that arise from $M_{12}$ in the manner we have just described. Thirdly, note that the given expressions (5.15) and (5.21) for the elliptic genera considered in this section point to explicit realizations in terms of the vertex algebra attached to the lattice $\mathbb{Z} \oplus(-\mathbb{Z}) \oplus\left(-E_{8}\right)$. It would be interesting to determine if the twinings (5.20) and (5.25) by elements of $M_{12}$ can also be realized using this structure. In view of the wellknown Mathieu moonshine connection between $M_{24}$ and K3 surfaces [2], it is appealing that the Euler characteristic of a half-K3 surface is 12 . So finally we ask, to what extent is the connection between half-K3 surfaces and $M_{12}$ described in this section related to the original Mathieu moonshine?

\section{Acknowledgments}

We are grateful to A. Klemm and J. Manschot for useful correspondence, and to G. Moore for helpful discussions and comments on an earlier draft. We would like to thank the American Institute of Mathematics for hosting four of us (MC, JD, SH and SK) as members of a SQuaRE working group on "Moonshine and String Theory" in May of 2017. Four of us (MC, JH, SH, and SK) also acknowledge the kind hospitality of the Aspen Center for Physics, which is supported by NSF grant PHY-1066293, as this was being completed. The work of M.C. was supported by ERC starting grant H2020 ERC StG \#640159. J.D. acknowledges support from the U.S. National Science Foundation (DMS 1601306), and the Simons Foundation (\#316779). J.H. acknowledges support from the U.S. National Science Foundation (PHY 1520748) and from the Simons Foundation (\#399639). S.H. is supported by a Harvard University Golub Fellowship in the Physical Sciences and DOE grant DE-SC0007870. S.K. is partially supported by a Simons Investigator Award and by the U.S. National Science Foundation under grant PHY-1720397.

\section{A New Mathieu moonshine}

Here we present numerical data in support of the discussions of section 5.2 and section 5.3 relating the M5-brane elliptic genera for degree one del Pezzo surfaces and half-K3 surfaces to the Mathieu group $M_{12}$. Since the relationship to half-K3 surfaces is formulated in terms of the functions that appear in section 5.2 we employ the notation of section 5.2 in what follows.

The character table of $M_{12}$ is table 1 , wherein $b_{11}:=-\frac{1}{2}+\frac{\sqrt{-11}}{2}$. Tables $2-3$ give the coefficients of $q^{d}$ in the McKay-Thompson series $f_{\mathrm{dP}_{8}, n Z}^{(1)}$ up to $d=\frac{2275}{24}$. The naming of the conjugacy classes is as in table 1 . Tables $4-8$ give the multiplicity generating functions for irreducible characters in the graded virtual $M_{12}$-module $W_{\mathrm{dP}_{8}, d}^{(1)}$ of (5.8). That is, for $\chi$ an irreducible character of $M_{12}$, the coefficient of $q^{d}$ in $f_{\mathrm{dP}_{8}, \chi}^{(1)}$ denotes the multiplicity 


\begin{tabular}{|c|rrrrrrrrrrrrrrr|}
\hline$[g]$ & $1 \mathrm{~A}$ & $2 \mathrm{~A}$ & $2 \mathrm{~B}$ & $3 \mathrm{~A}$ & $3 \mathrm{~B}$ & $4 \mathrm{~A}$ & $4 \mathrm{~B}$ & $5 \mathrm{~A}$ & $6 \mathrm{~A}$ & $6 \mathrm{~B}$ & $8 \mathrm{~A}$ & $8 \mathrm{~B}$ & $10 \mathrm{~A}$ & $11 \mathrm{~A}$ & $11 \mathrm{~B}$ \\
\hline$n \mid h$ & $1 \mid 1$ & $2 \mid 2$ & $2 \mid 1$ & $3 \mid 1$ & $3 \mid 3$ & $4 \mid 1$ & $4 \mid 1$ & $5 \mid 1$ & $6 \mid 6$ & $6 \mid 1$ & $8 \mid 1$ & $8 \mid 1$ & $10 \mid 2$ & $11 \mid 1$ & $11 \mid 1$ \\
\hline$\chi_{1}$ & 1 & 1 & 1 & 1 & 1 & 1 & 1 & 1 & 1 & 1 & 1 & 1 & 1 & 1 & 1 \\
$\chi_{2}$ & 11 & -1 & 3 & 2 & -1 & -1 & 3 & 1 & -1 & 0 & -1 & 1 & -1 & 0 & 0 \\
$\chi_{3}$ & 11 & -1 & 3 & 2 & -1 & 3 & -1 & 1 & -1 & 0 & 1 & -1 & -1 & 0 & 0 \\
$\chi_{4}$ & 16 & 4 & 0 & -2 & 1 & 0 & 0 & 1 & 1 & 0 & 0 & 0 & -1 & $b_{11}$ & $\overline{b_{11}}$ \\
$\chi_{5}$ & 16 & 4 & 0 & -2 & 1 & 0 & 0 & 1 & 1 & 0 & 0 & 0 & -1 & $\overline{b_{11}}$ & $b_{11}$ \\
$\chi_{6}$ & 45 & 5 & -3 & 0 & 3 & 1 & 1 & 0 & -1 & 0 & -1 & -1 & 0 & 1 & 1 \\
$\chi_{7}$ & 54 & 6 & 6 & 0 & 0 & 2 & 2 & -1 & 0 & 0 & 0 & 0 & 1 & -1 & -1 \\
$\chi_{8}$ & 55 & -5 & 7 & 1 & 1 & -1 & -1 & 0 & 1 & 1 & -1 & -1 & 0 & 0 & 0 \\
$\chi_{9}$ & 55 & -5 & -1 & 1 & 1 & 3 & -1 & 0 & 1 & -1 & -1 & 1 & 0 & 0 & 0 \\
$\chi_{10}$ & 55 & -5 & -1 & 1 & 1 & -1 & 3 & 0 & 1 & -1 & 1 & -1 & 0 & 0 & 0 \\
$\chi_{11}$ & 66 & 6 & 2 & 3 & 0 & -2 & -2 & 1 & 0 & -1 & 0 & 0 & 1 & 0 & 0 \\
$\chi_{12}$ & 99 & -1 & 3 & 0 & 3 & -1 & -1 & -1 & -1 & 0 & 1 & 1 & -1 & 0 & 0 \\
$\chi_{13}$ & 120 & 0 & -8 & 3 & 0 & 0 & 0 & 0 & 0 & 1 & 0 & 0 & 0 & -1 & -1 \\
$\chi_{14}$ & 144 & 4 & 0 & 0 & -3 & 0 & 0 & -1 & 1 & 0 & 0 & 0 & -1 & 1 & 1 \\
$\chi_{15}$ & 176 & -4 & 0 & -4 & -1 & 0 & 0 & 1 & -1 & 0 & 0 & 0 & 1 & 0 & 0 \\
\hline
\end{tabular}

Table 1. Character table of $M_{12}$.

of $\chi$ in the (virtual) $M_{12}$-module $W_{\mathrm{dP}_{8}, d}^{(1)}$. In tables 4-8 the characters are named by their dimensions, and appear in the same order as in table 1.

The modular forms $f_{\mathrm{dP}_{8}, n Z}^{(1)}$ may be realized as Rademacher sums. Specifically, consider the degree 24 permutation representation of $M_{12}$ that arises by restricting the defining permutation representation of $M_{24}$. The corresponding character is $2 \chi_{1}+\chi_{2}+\chi_{3}$ in the notation of table 1. If $g \in M_{12}$ has order $n$ and $h$ is the minimal length of a cycle in the cycle shape of $g$ (in this permutation representation) then $f_{\mathrm{dP}_{8},[g]}^{(1)}$ is the Rademacher sum of weight $\frac{3}{2}$ for $\Gamma_{0}(n)$ with polar part $q^{-\frac{5}{24}}$ (at the infinite cusp), and multiplier system given by $\gamma \mapsto \mathrm{e}\left(\frac{c d}{n h}\right) \epsilon^{-5}(\gamma)$ for $\gamma=\left(\begin{array}{ll}* * \\ c & d\end{array}\right) \in \Gamma_{0}(n)$, where $\epsilon$ is the multiplier system of $\eta$. The values of $n$ and $h$ for each conjugacy class $[g] \subset M_{12}$ are given in table 1 . We refer to [37] for details of the Rademacher sum construction.

Note that since the factor $\mathrm{e}\left(\frac{c d}{n h}\right)$ is trivial on $\Gamma_{0}(n h)$ the function $f_{\mathrm{dP}_{8},[g]}^{(1)} \eta^{5}$ is a holomorphic modular form of weight 4 for $\Gamma_{0}(n h)$. This, together with the Fourier coefficients in tables 2-3 gives an alternative method for reconstructing the $f_{\mathrm{dP}_{8}, n Z}^{(1)}$. For example, we find in this way that

$$
\begin{aligned}
f_{\mathrm{dP}_{8}, 2 \mathrm{~B}}^{(1)}(\tau) & =\frac{1}{15}\left(16 E_{4}(2 \tau)-E_{4}(\tau)\right) \eta^{-5}(\tau), \\
f_{\mathrm{dP}_{8}, 3 \mathrm{~A}}^{(1)}(\tau) & =\frac{1}{80}\left(81 E_{4}(3 \tau)-E_{4}(\tau)\right) \eta^{-5}(\tau), \\
f_{\mathrm{dP}_{8}, 2 \mathrm{~A}}^{(1)}(\tau) & =f_{\mathrm{dP}_{8}, 2 B}^{(1)}(\tau)+32 \eta^{3}(\tau) \eta(4 \tau)^{8} \eta^{-8}(2 \tau) .
\end{aligned}
$$




\begin{tabular}{|c|c|c|c|c|c|c|c|c|c|c|c|c|}
\hline $24 d$ & $1 \mathrm{~A}$ & $2 \mathrm{~A}$ & $2 \mathrm{~B}$ & $3 \mathrm{~A}$ & $3 B$ & $4 \mathrm{AB}$ & $5 \mathrm{~A}$ & $6 \mathrm{~A}$ & $6 \mathrm{~B}$ & $8 \mathrm{AB}$ & $10 \mathrm{~A}$ & $11 \mathrm{AB}$ \\
\hline-5 & 1 & 1 & 1 & 1 & 1 & 1 & 1 & 1 & 1 & 1 & 1 & 1 \\
\hline 19 & 245 & 21 & -11 & 2 & -7 & 5 & -5 & -3 & -2 & 1 & 1 & 3 \\
\hline 43 & 3380 & -44 & 52 & -22 & 14 & 4 & 5 & -2 & -2 & 0 & 1 & -8 \\
\hline 67 & 22385 & 113 & -143 & 29 & 11 & -15 & 10 & 11 & 1 & 1 & -2 & 0 \\
\hline 91 & 110110 & -322 & 286 & 31 & -77 & -18 & -15 & -1 & 7 & -6 & -7 & 0 \\
\hline 115 & 438746 & 602 & -550 & -112 & 77 & 26 & -4 & -7 & 0 & -6 & 2 & 0 \\
\hline 139 & 1531985 & -1071 & 1105 & 113 & 77 & 17 & -15 & -3 & -11 & 1 & -1 & 4 \\
\hline 163 & 4804910 & 1870 & -2002 & 71 & -253 & -66 & 35 & -17 & -13 & -6 & -5 & 0 \\
\hline 187 & 13914285 & -3283 & 3245 & -381 & 231 & -19 & 35 & 11 & -13 & 13 & 7 & 11 \\
\hline 211 & 37674325 & 5525 & -5291 & 334 & 145 & 117 & -50 & 29 & 10 & 13 & 0 & 7 \\
\hline 235 & 96580627 & -8621 & 8723 & 277 & -704 & 51 & 2 & 16 & 29 & -5 & 4 & 0 \\
\hline 259 & 236144545 & 13377 & -13663 & -911 & 637 & -143 & -80 & -15 & 17 & 13 & 12 & -23 \\
\hline 283 & 4578570 & -20790 & 20618 & 811 & 469 & -86 & 70 & -39 & -1 & -14 & 10 & -4 \\
\hline 307 & 1256789730 & 31458 & -31006 & 534 & -1617 & 226 & 105 & -21 & -46 & -14 & -7 & -2 \\
\hline 331 & 2760379655 & -46073 & 46343 & -2173 & 1364 & 135 & -95 & 4 & -25 & 7 & -13 & 0 \\
\hline 355 & 5894771883 & 67179 & -67925 & 1824 & 924 & -373 & 8 & 48 & 16 & -13 & 4 & 0 \\
\hline 379 & 12275038600 & -97752 & 97416 & 1249 & -3575 & -168 & -150 & 33 & 45 & 20 & -12 & 0 \\
\hline 403 & 982062560 & 139584 & -138528 & -4420 & 2915 & 528 & 185 & -9 & 36 & 20 & -11 & -7 \\
\hline 427 & 794727675 & -196133 & 196603 & 3595 & 2002 & 235 & 175 & -62 & -29 & -1 & 7 & 0 \\
\hline 451 & 369902630 & 274022 & -275418 & 2376 & -6930 & -698 & -245 & -46 & -72 & 14 & 7 & 11 \\
\hline 475 & 187076653120 & -381024 & 380224 & -8738 & 5698 & -400 & -5 & 42 & -62 & -44 & 1 & 38 \\
\hline 499 & 16436085 & 524277 & -522379 & 7079 & 3641 & 949 & -290 & 81 & 35 & -43 & 12 & 0 \\
\hline 523 & 8376681690 & -713734 & 714714 & 4727 & -13300 & 490 & 315 & 80 & 123 & 6 & 11 & -31 \\
\hline 547 & 1208616966765 & 966093 & -968851 & -16155 & 10593 & -1379 & 390 & -51 & 89 & -31 & -22 & 32 \\
\hline 571 & 2189664565985 & -1302879 & 1301729 & 12908 & 7004 & -575 & -390 & -144 & -40 & 57 & -24 & -27 \\
\hline 595 & 18286118747 & 155 & -1740453 & 8147 & -23947 & 1851 & -3 & -111 & -141 & 51 & 5 & 0 \\
\hline 619 & 64880 & 048 & 2317744 & -29150 & 18865 & 848 & -495 & 45 & -98 & -24 & -13 & 0 \\
\hline 643 & 12125024699095 & 3062199 & -3066921 & 22825 & 35 & -2361 & 595 & 183 & 45 & 43 & -21 & 0 \\
\hline 667 & 20994476982895 & -4034001 & 599 & 14815 & -42092 & -1201 & 645 & 132 & 175 & -57 & 29 & -24 \\
\hline 691 & 35997712990855 & 5283495 & -5277305 & -50087 & 32956 & 3095 & -770 & -60 & 133 & -45 & 20 & 0 \\
\hline 715 & 61152257 & -6880923 & 6883877 & 39062 & 98 & 1477 & -14 & -234 & -106 & 13 & 2 & 11 \\
\hline 739 & 102971911295570 & 122 & -8935342 & 24530 & -71194 & -4110 & -930 & -134 & 50 & -38 & 32 & 42 \\
\hline 763 & 171940936021855 & -11542209 & 11538527 & -84881 & 32 & -1841 & 980 & 120 & -193 & 83 & 36 & 0 \\
\hline 787 & 284816074366495 & 431 & -14847713 & 65539 & 34552 & 5359 & 1120 & 280 & 127 & 75 & -24 & -54 \\
\hline 811 & 468201092136435 & -19041645 & 19046643 & 41319 & 118503 & 2499 & -1190 & 213 & 339 & -1 & -20 & 31 \\
\hline 835 & 764062908896885 & 320821 & -24334219 & -138991 & 923 & 699 & 10 & -125 & 233 & 45 & -4 & -51 \\
\hline 859 & 1238199118586430 & -30972578 & 30966078 & 106548 & 57057 & -3250 & -1445 & -407 & -96 & -118 & -43 & 0 \\
\hline 883 & 1993162922073180 & 39301308 & -39284388 & 66213 & -191268 & 8460 & 1680 & -264 & -423 & -112 & -32 & 0 \\
\hline 907 & 3187894582604875 & -49688021 & 49696075 & -224249 & 146146 & 4027 & 1750 & 118 & -269 & 31 & 34 & 0 \\
\hline 931 & 5067385763330905 & 62640249 & -62662055 & 170881 & 90349 & -10903 & -1970 & 477 & 157 & -75 & 44 & -8 \\
\hline 955 & 8007296783387517 & -78760899 & 78751101 & 106299 & -304920 & -4899 & 17 & 324 & 507 & 149 & 1 & 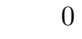 \\
\hline 979 & 12580745731759205 & 98730149 & -98702747 & -353800 & 231308 & 13701 & -2420 & -196 & 352 & 125 & 44 & 55 \\
\hline 1003 & 19657853187268125 & -123390883 & 123403293 & 268005 & 143418 & 6205 & 2500 & -574 & -243 & -43 & 32 & 93 \\
\hline 1027 & 30553454673736995 & 153823299 & -153856989 & 164577 & -475629 & -16845 & 2870 & -429 & -639 & 111 & -46 & 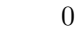 \\
\hline 1051 & 47245393529211705 & -191308807 & 191292729 & -550938 & 359007 & -8039 & -3045 & 299 & -462 & -175 & -67 & -96 \\
\hline 1075 & 72695798459621870 & 237317774 & -237275922 & 414887 & 219776 & 20926 & -5 & 752 & 267 & -150 & -1 & 38 \\
\hline 1099 & 111322113952614145 & -293640095 & 293659649 & 255274 & -732809 & 9777 & -3605 & 559 & 818 & 37 & -45 & -57 \\
\hline 1123 & 169685042685799025 & 362516273 & -362568591 & -842182 & 550550 & -26159 & 4025 & -310 & 558 & -119 & -67 & 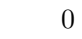 \\
\hline
\end{tabular}

Table 2. McKay-Thompson series $f_{\mathrm{dP}_{8}, n Z}^{(1)}$, part 1 . 


\begin{tabular}{|c|c|c|c|c|c|c|c|c|c|c|c|c|}
\hline $24 d$ & $1 \mathrm{~A}$ & $2 \mathrm{~A}$ & $2 \mathrm{~B}$ & $3 \mathrm{~A}$ & $3 \mathrm{~B}$ & $4 \mathrm{AB}$ & $5 \mathrm{~A}$ & $6 \mathrm{~A}$ & $6 \mathrm{~B}$ & $8 \mathrm{AB}$ & $10 \mathrm{~A}$ & $11 \mathrm{AB}$ \\
\hline 1147 & 257489647589909735 & -446610297 & 446586855 & 631943 & 337337 & -11721 & 4235 & -963 & -273 & 195 & 63 & 0 \\
\hline 1171 & 389036935666670005 & 548983797 & -548919371 & 384994 & -1110824 & 32213 & -4745 & -648 & -1010 & 189 & 57 & 0 \\
\hline 1195 & 585321427870953137 & -673325583 & 673354929 & -1274347 & 830753 & 14673 & 12 & 333 & -699 & -39 & -8 & 1 \\
\hline 1219 & 877051946642599585 & 824194401 & -824272735 & 951751 & 505153 & -39167 & -5540 & 1125 & 383 & 121 & 56 & 0 \\
\hline 1243 & 1308987868412927175 & -1006964089 & 1006927559 & 579426 & -1666434 & -18265 & 5925 & 770 & 1178 & -273 & 81 & 66 \\
\hline 1267 & 1946143827213299420 & 1227840124 & -1227744804 & -1900084 & 1241051 & 47660 & 6545 & -413 & 852 & -248 & -71 & 80 \\
\hline 1291 & 2882640700620305625 & -1494261447 & 1494305241 & 1413606 & 753753 & 21897 & -6875 & -1347 & -534 & 45 & -57 & 0 \\
\hline 1315 & 4254293388075663716 & 1815256100 & -1815372956 & 855485 & -2465848 & -58428 & -34 & -964 & -1451 & -180 & 0 & -151 \\
\hline 1339 & 6256460810550333175 & -2201441577 & 2201389047 & -2806274 & 1830877 & -26265 & -8075 & 573 & -1026 & 347 & -87 & 34 \\
\hline 1363 & 9169285487540907855 & 2665121487 & -2664979633 & 2080578 & 1105104 & 70927 & 8855 & 1656 & 602 & 303 & -73 & -105 \\
\hline 1387 & 13393282867503977295 & -3220900017 & 3220964175 & 1257615 & -3616767 & 32079 & 9420 & 1173 & 1839 & -97 & 68 & 0 \\
\hline 1411 & 19499387894072616035 & 3886321059 & -3886491037 & -4096807 & 2675288 & -84989 & -10340 & -708 & 1265 & 235 & 104 & 0 \\
\hline 1435 & 28299145776890952502 & -4681963594 & 4681885494 & 3027835 & 1613458 & -39050 & 2 & -2050 & -645 & -362 & 6 & 0 \\
\hline 1459 & 40942900182985321885 & 5631643773 & -5631439203 & 1820005 & -5246318 & 102285 & -11990 & -1398 & -2151 & -319 & 88 & -32 \\
\hline 1483 & 59056800250327174295 & -6763471561 & 6763564951 & -5930560 & 3868865 & 46695 & 12670 & 713 & -1460 & 99 & 94 & 0 \\
\hline 1507 & 84933501203533915790 & 8110930190 & -8111177074 & 4368671 & 2321594 & -123442 & 13790 & 2474 & 791 & -242 & -110 & 121 \\
\hline 1531 & 121796962409191079920 & -9713117456 & 9713006576 & 2623747 & -7548422 & -55440 & -14705 & 1666 & 2519 & 448 & -111 & 184 \\
\hline 1555 & 174169266048720253146 & 11615262618 & -11614967078 & -8500497 & 5550897 & 147770 & 21 & -891 & 1759 & 386 & -7 & 0 \\
\hline 1579 & 248377592707632513150 & -13870493250 & 13870626430 & 6245646 & 3326730 & 66590 & -16850 & -2850 & -1094 & -58 & -100 & -206 \\
\hline 1603 & 353253324430055413910 & 16541557942 & -16541909098 & 3734813 & -10760596 & -175578 & 18410 & -1988 & -3067 & 274 & -118 & 106 \\
\hline 1627 & 501093959691581866120 & -19701491768 & 19701331592 & -12095267 & 7891807 & -80088 & 19495 & 1231 & -2191 & -576 & 147 & -160 \\
\hline 1651 & 708983776598335157920 & 23434722304 & -23434304608 & 8862610 & 4711630 & 208848 & -21080 & 3418 & 1250 & -524 & 124 & 0 \\
\hline 1675 & 1000603206531275493740 & -27839878548 & 27840067948 & 5292041 & -15230776 & 94700 & -10 & 2424 & 3709 & 124 & 2 & 0 \\
\hline 1699 & 1408702624118535140395 & 33032707435 & -33033205205 & -17064938 & 11141053 & -248885 & -24230 & -1379 & 2530 & -365 & 160 & 0 \\
\hline 1723 & 1978477667607266537870 & -39147551762 & 39147329166 & 12473543 & 6641696 & -111298 & 25620 & -4172 & -1317 & 674 & 148 & -7 \\
\hline 1747 & 2772165465354178800390 & 46339376966 & -46338787066 & 7423860 & -21384528 & 294950 & 27765 & -2872 & -4360 & 606 & -139 & 0 \\
\hline 1771 & 3875291180776298187905 & -54788436927 & 54788701825 & -23916919 & 15607823 & 132449 & -29345 & 1491 & -2951 & -183 & -137 & 121 \\
\hline 1795 & 5405141185164404628126 & 64705171230 & -64705866082 & 17441601 & 9274839 & -347426 & 1 & 4887 & 1649 & 462 & 5 & 226 \\
\hline 1819 & 7522234988258726535800 & -76332974536 & 76332659832 & 10365428 & -29834728 & -157352 & -33575 & 3284 & 5076 & -768 & -191 & 0 \\
\hline 1843 & 10445828606352623315780 & 89952081860 & -89951262908 & -33278401 & 21724703 & 409476 & 36155 & -1885 & 3559 & -652 & -165 & -261 \\
\hline 1867 & 14474828224085044461275 & -105887271301 & 105887640027 & 24216572 & 12892502 & 184363 & 38150 & -5626 & -2112 & 167 & 154 & 124 \\
\hline 1891 & 20015952136902352017085 & 124515746461 & -124516712515 & 14350090 & -41334620 & -483027 & -41165 & -3956 & -5998 & -495 & 191 & -203 \\
\hline 1915 & 27621587036170435738716 & -146272523812 & 146272092252 & -46027323 & 30036996 & -215780 & -34 & 2336 & -4179 & 892 & -12 & 0 \\
\hline 1939 & 38040588686504509649390 & 171657529358 & -171656394514 & 33423194 & 17775758 & 567422 & -46735 & 6710 & 2378 & 810 & 173 & 0 \\
\hline 1963 & 52286338577292264775330 & -201247917374 & 201248425378 & 19779820 & -56935802 & 254002 & 49455 & 4666 & 7156 & -162 & 171 & 0 \\
\hline 1987 & 71727767470164471985970 & 235711210322 & -235712537038 & -63253216 & 41292251 & -663358 & 53095 & -2629 & 4868 & 558 & -213 & -75 \\
\hline 2011 & 98210898877425806480580 & -275815152540 & 275814555588 & 45846324 & 24403500 & -298476 & -56045 & -7932 & -2592 & -1104 & -215 & 0 \\
\hline 2035 & 134220886793221405680657 & 322440005713 & -322438454767 & 27064422 & -77947254 & 775473 & 32 & -5402 & -8362 & -999 & -12 & 187 \\
\hline 2059 & 183097700277061507239685 & -376598728955 & 376599424773 & -86460161 & 56425369 & 347909 & -63565 & 2905 & -5649 & 229 & -205 & 328 \\
\hline 2083 & 249322773322218559282605 & 439458518285 & -439460332883 & 62548884 & 33271029 & -907299 & 67980 & 9221 & 3160 & -703 & -240 & 0 \\
\hline 2107 & 338899391506589069707460 & -512358042588 & 512357234116 & 36873998 & -106155721 & -404236 & 71960 & 6279 & 9562 & 1288 & 252 & -399 \\
\hline 2131 & 459856714942619762629680 & 596828898736 & -596826783696 & -117510219 & 76706784 & 1057520 & -76945 & -3464 & 6585 & 1104 & 241 & 203 \\
\hline 2155 & 622916642534098871009415 & -694628315129 & 694629258375 & 84864729 & 45168123 & 471623 & 40 & -10613 & -3831 & -313 & -4 & -329 \\
\hline 2179 & 842374854253122861597685 & 807773972437 & -807776428811 & 49926859 & -143781275 & -1228187 & -86815 & -7307 & -11201 & 825 & 247 & 0 \\
\hline 2203 & 1137263164627672249293665 & -938574065919 & 938572965473 & -158926840 & 103724390 & -550223 & 91665 & 4242 & -7780 & -1427 & 301 & 0 \\
\hline 2227 & 1532880862104682603160505 & 1089663943033 & -1089661090375 & 114584796 & 60954696 & 1426329 & 97755 & 12388 & 4364 & -1247 & -257 & 0 \\
\hline 2251 & 2062809388122724224605390 & -1264058425906 & 1264059700430 & 67322939 & -193818955 & 637262 & -103235 & 8561 & 13199 & 302 & -261 & -67 \\
\hline 2275 & 2771559316831560463943735 & 1465208427543 & -1465211739849 & -213852964 & 139592453 & -1656153 & -15 & -4851 & 9012 & -901 & -7 & 0 \\
\hline
\end{tabular}

Table 3. McKay-Thompson series $f_{\mathrm{dP}_{8}, n Z}^{(1)}$, part 2 . 


\begin{tabular}{|c|c|c|c|c|c|c|c|c|}
\hline $24 d$ & 1 & 11 & $11^{\prime}$ & 16 & $16^{\prime}$ & 45 & 54 & 55 \\
\hline-5 & 1 & 0 & 0 & 0 & 0 & 0 & 0 & 0 \\
\hline 19 & 0 & 0 & 0 & -1 & -1 & 1 & 1 & -2 \\
\hline 43 & -1 & 1 & 1 & 2 & 2 & 0 & 4 & 4 \\
\hline 67 & 2 & 0 & 0 & 7 & 7 & 14 & 8 & 8 \\
\hline 91 & -4 & 20 & 20 & 9 & 9 & 35 & 62 & 83 \\
\hline 115 & 5 & 35 & 35 & 89 & 89 & 239 & 251 & 222 \\
\hline 139 & 20 & 201 & 201 & 236 & 236 & 694 & 881 & 950 \\
\hline 163 & 36 & 528 & 528 & 833 & 833 & 2323 & 2702 & 2665 \\
\hline 187 & 156 & 1655 & 1655 & 2311 & 2311 & 6485 & 7918 & 8235 \\
\hline 211 & 413 & 4263 & 4263 & 6423 & 6423 & 18051 & 21397 & 21498 \\
\hline 235 & 1020 & 11382 & 11382 & 16087 & 16087 & 45358 & 54939 & 56379 \\
\hline 259 & 2455 & 26994 & 26994 & 40021 & 40021 & 112341 & 134076 & 135889 \\
\hline 283 & 5879 & 64617 & 64617 & 93003 & 93003 & 261869 & 315210 & 322155 \\
\hline 307 & 13169 & 144938 & 144938 & 212050 & 212050 & 596095 & 713920 & 725468 \\
\hline 331 & 29087 & 320286 & 320286 & 464053 & 464053 & 1305434 & 1568719 & 1600075 \\
\hline 355 & 61991 & 680939 & 680939 & 993468 & 993468 & 2793599 & 3348812 & 3407543 \\
\hline 379 & 129169 & 1422770 & 1422770 & 2064719 & 2064719 & 5808170 & 6975047 & 7109138 \\
\hline 403 & 262777 & 2888505 & 2888505 & 4208325 & 4208325 & 11833971 & 14193562 & 14449220 \\
\hline 427 & 524286 & 5767282 & 5767282 & 8379616 & 8379616 & 23570078 & 28293712 & 28827745 \\
\hline 451 & 1023996 & 11264435 & 11264435 & 16396496 & 16396496 & 46112567 & 55321988 & 56332449 \\
\hline 475 & 1968752 & 21659408 & 21659408 & 31488517 & 31488517 & 88564546 & 106295853 & 108283778 \\
\hline 499 & 3720441 & 40917665 & 40917665 & 59539940 & 59539940 & 167451476 & 200915361 & 204609370 \\
\hline 523 & 6927917 & 76215745 & 76215745 & 110825425 & 110825425 & 311704255 & 374082188 & 381045664 \\
\hline 547 & 12715863 & 139866126 & 139866126 & 203487898 & 203487898 & 572297901 & 686707842 & 699375765 \\
\hline 571 & 23041098 & 253459390 & 253459390 & 368608354 & 368608354 & 1036726053 & 1244135671 & 1267242033 \\
\hline 595 & 41225542 & 453471967 & 453471967 & 659672220 & 659672220 & 1855310757 & 2226288378 & 2267426223 \\
\hline 619 & 72924892 & 802191793 & 802191793 & 1166723003 & 1166723003 & 3281429787 & 3937829833 & 4010870330 \\
\hline 643 & 127575605 & 1403299073 & 1403299073 & 2041300549 & 2041300549 & 5741128020 & 6889198936 & 7016622004 \\
\hline 667 & 220904792 & 2429997813 & 2429997813 & 3534354858 & 3534354858 & 9940416134 & 11928705034 & 12149811849 \\
\hline 691 & 378758456 & 4166294843 & 4166294843 & 6060311787 & 6060311787 & 17044567168 & 20453213655 & 20831707181 \\
\hline 715 & 643445607 & 7077944811 & 7077944811 & 10294877321 & 10294877321 & 28954418447 & 34745644272 & 35389433549 \\
\hline 739 & 1083447396 & 11917871022 & 11917871022 & 17335484390 & 17335484390 & 48755959253 & 58506711305 & 59589713385 \\
\hline 763 & 1809154794 & 19900794975 & 19900794975 & 28946097935 & 28946097935 & 81411011737 & 97693785348 & 99503517664 \\
\hline 787 & 2996789379 & 32964531410 & 32964531410 & 47949080384 & 47949080384 & 134856640526 & 161827223016 & 164823268101 \\
\hline 811 & 4926375742 & 54190323007 & 54190323007 & 78821410398 & 78821410398 & 221685419135 & 266023467395 & 270950794599 \\
\hline 835 & 8039356619 & 88432717409 & 88432717409 & 128630532359 & 128630532359 & 361773104706 & 434126499529 & 442164641760 \\
\hline 859 & 13028224735 & 143310698119 & 143310698119 & 208450511320 & 208450511320 & 586267397566 & 703522419579 & 716552193823 \\
\hline 883 & 20971787728 & 230689383931 & 230689383931 & 335549960886 & 335549960886 & 943733861399 & 1132478688778 & 1153448509388 \\
\hline 907 & 33542713964 & 368970251977 & 368970251977 & 536681774077 & 536681774077 & 1509417984175 & 1811304051250 & 1844849248586 \\
\hline 931 & 53318392379 & 586501744668 & 586501744668 & 853096286376 & 853096286376 & 2399332671290 & 2879196063095 & 2932511326216 \\
\hline 955 & 84251935429 & 926772031772 & 926772031772 & 1348028436453 & 1348028436453 & 3791330807137 & 4549600936442 & 4633856811066 \\
\hline 979 & 132373064244 & 1456102857556 & 1456102857556 & 2117972326676 & 2117972326676 & 5956796105864 & 7148150369696 & 7280518493936 \\
\hline 1003 & 206837818945 & 2275216938197 & 2275216938197 & 3309400831213 & 3309400831213 & 9307691151506 & 11169235537586 & 11376079524539 \\
\hline 1027 & 321479780918 & 3536276449763 & 3536276449763 & 5143681782673 & 5143681782673 & 14466603424870 & 17359916463414 & 17681388557332 \\
\hline 1051 & 497110820190 & 5468220598525 & 5468220598525 & 7953766796046 & 7953766796046 & 22369971051653 & 26843974790631 & 27341095178372 \\
\hline 1075 & 764896637498 & 8413860876702 & 8413860876702 & 12238353855121 & 12238353855121 & 34420367790300 & 41304429463648 & 42069314230101 \\
\hline 1099 & 1171318829840 & 12884509771652 & 12884509771652 & 18741091700903 & 18741091700903 & 52709323491410 & 63251202946890 & 64422536456281 \\
\hline 1123 & 1785406214518 & 19639465309775 & 19639465309775 & 28566511591466 & 28566511591466 & 80343309988087 & 96411953800436 & 98197341895753 \\
\hline 1147 & 2709277080802 & 29802051397724 & 29802051397724 & 43348418062984 & 43348418062984 & 121917430520635 & 146300938919330 & 149010238333467 \\
\hline
\end{tabular}

Table 4. Multiplicity generating functions $f_{\mathrm{dP}_{8}, \chi}^{(1)}$, part 1 . 


\begin{tabular}{|c|c|c|c|c|c|c|c|}
\hline $24 d$ & $55^{\prime}$ & $55^{\prime \prime}$ & 66 & 99 & 120 & 144 & 176 \\
\hline-5 & 0 & 0 & 0 & 0 & 0 & 0 & 0 \\
\hline 19 & 0 & 0 & 0 & 0 & 0 & 2 & 0 \\
\hline 43 & 3 & 3 & 1 & 5 & 2 & 1 & 9 \\
\hline 67 & 12 & 12 & 21 & 21 & 36 & 35 & 37 \\
\hline 91 & 65 & 65 & 72 & 116 & 130 & 170 & 207 \\
\hline 115 & 244 & 244 & 303 & 450 & 572 & 668 & 809 \\
\hline 139 & 910 & 910 & 1053 & 1625 & 1892 & 2299 & 2843 \\
\hline 163 & 2743 & 2743 & 3380 & 4946 & 6152 & 7328 & 8873 \\
\hline 187 & 8105 & 8105 & 9602 & 14577 & 17408 & 21007 & 25847 \\
\hline 211 & 21733 & 21733 & 26243 & 39149 & 47808 & 57171 & 69639 \\
\hline 235 & 56011 & 56011 & 66950 & 100712 & 121602 & 146250 & 178995 \\
\hline 259 & 136438 & 136438 & 164139 & 245788 & 298688 & 357966 & 437126 \\
\hline 283 & 321282 & 321282 & 384883 & 578131 & 699412 & 839873 & 1027282 \\
\hline 307 & 726800 & 726800 & 873253 & 1308380 & 1588170 & 1904874 & 2326881 \\
\hline 331 & 1598171 & 1598171 & 1916117 & 2876429 & 3483272 & 4181523 & 5112694 \\
\hline 355 & 3410318 & 3410318 & 4094710 & 6139138 & 7445828 & 8932518 & 10914961 \\
\hline 379 & 7105048 & 7105048 & 8522970 & 12788159 & 15494804 & 18597231 & 22733170 \\
\hline 403 & 14455051 & 14455051 & 17350401 & 26020434 & 31548542 & 37853674 & 46261017 \\
\hline 427 & 28819592 & 28819592 & 34577010 & 51873536 & 62864134 & 75443098 & 92215429 \\
\hline 451 & 56343865 & 56343865 & 67622177 & 101421034 & 122953390 & 147535322 & 180310064 \\
\hline 475 & 108267895 & 108267895 & 129908352 & 194879528 & 236191550 & 283442660 & 346445082 \\
\hline 499 & 204631232 & 204631232 & 245574876 & 368340362 & 446507564 & 535790948 & 654835854 \\
\hline 523 & 381015906 & 381015906 & 457195844 & 685822008 & 831254198 & 997529608 & 1219228018 \\
\hline 547 & 699415924 & 699415924 & 839330695 & 1258957772 & 1526071004 & 1831253012 & 2238164410 \\
\hline 571 & 1267187750 & 1267187750 & 1520582135 & 2280927045 & 2764674464 & 3317651311 & 4054954916 \\
\hline 595 & 2267499033 & 2267499033 & 2721057746 & 4081511489 & 4947403906 & 5936828205 & 7256056407 \\
\hline 619 & 4010773890 & 4010773890 & 4812849666 & 7219375201 & 8750602126 & 10500800283 & 12834400672 \\
\hline 643 & 7016749493 & 7016749493 & 8420202262 & 12630174462 & 15309503684 & 18371299544 & 22453696426 \\
\hline 667 & 12149643643 & 12149643643 & 14579440062 & 21869323134 & 26508010882 & 31809749824 & 38878728441 \\
\hline 691 & 20831927400 & 20831927400 & 24998485648 & 37497515848 & 45451874944 & 54542074762 & 66662346131 \\
\hline 715 & 35389146944 & 35389146944 & 42466747340 & 63700406402 & 77212161966 & 92654819516 & 113245032965 \\
\hline 739 & 59590085251 & 59590085251 & 71508403663 & 107262225204 & 130015413134 & 156018202222 & 190688575918 \\
\hline 763 & 99503036747 & 99503036747 & 119403255079 & 179105374690 & 217096655922 & 260516372674 & 318409337999 \\
\hline 787 & 164823887402 & 164823887402 & 197789160389 & 296683119323 & 359616877840 & 431539751281 & 527436921384 \\
\hline 811 & 270950001188 & 270950001188 & 325139371484 & 487709837991 & 591162203858 & 709395286805 & 867039376965 \\
\hline 835 & 442165654782 & 442165654782 & 530599589906 & 795898389892 & 964726901324 & 1157671471836 & 1414930915206 \\
\hline 859 & 716550903166 & 716550903166 & 859860053460 & 1289791366536 & 1563381441138 & 1876058749736 & 2292961837333 \\
\hline 883 & 1153450147408 & 1153450147408 & 1384141494283 & 2076210582947 & 2516621491560 & 3019944492097 & 3691041793857 \\
\hline 907 & 1844847178517 & 1844847178517 & 2213814945093 & 3320724518828 & 4025117339308 & 4830142466496 & 5903509327159 \\
\hline 931 & 2932513935701 & 2932513935701 & 3519018815116 & 5278525605052 & 6398216968080 & 7677858253874 & 9384046650468 \\
\hline 955 & 4633853529026 & 4633853529026 & 5560621623978 & 8340935682923 & 10110219936042 & 12132266566371 & 14828328689896 \\
\hline 979 & 7280522608177 & 7280522608177 & 8736630398824 & 13104941535681 & 15884784057350 & 19061737583669 & 23297675655094 \\
\hline 1003 & 11376074383581 & 11376074383581 & 13651285150689 & 20476932857057 & 24820516574124 & 29784623972565 & 36403433861254 \\
\hline 1027 & 17681394966175 & 17681394966175 & 21217679111305 & 31826512201395 & 38577600704766 & 46293115745065 & 56580469055649 \\
\hline 1051 & 27341087206947 & 27341087206947 & 32809298242028 & 49213955409660 & 59653266656898 & 71583926371636 & 87491472717522 \\
\hline 1075 & 42069324119087 & 42069324119087 & 50483196856446 & 75724785382753 & 91787634227288 & 110145153118297 & 134621845007103 \\
\hline 1099 & 64422524221421 & 64422524221421 & 77307019308615 & 115960541117888 & 140558212466154 & 168669864792640 & 206152067770248 \\
\hline 1123 & 98197356999292 & 98197356999292 & 117836840441407 & 176755245669203 & 214248806330278 & 257098555519575 & 314231554534832 \\
\hline 1147 & 149010219724356 & 149010219724356 & 178812248794508 & 268218391773495 & 325113172828678 & 390135822209555 & 476832688109889 \\
\hline
\end{tabular}

Table 5. Multiplicity generating functions $f_{\mathrm{dP}_{8}, \chi}^{(1)}$, part 2 . 


\begin{tabular}{|c|c|c|c|c|c|}
\hline $24 d$ & 1 & 11 & $11^{\prime}$ & 16 & $16^{\prime}$ \\
\hline 1171 & 4093401506990 & 45027412291973 & 45027412291973 & 65494442717998 & 65494442717998 \\
\hline 1195 & 6158685759395 & 67745548890523 & 67745548890523 & 98538949769247 & 98538949769247 \\
\hline 1219 & 9228239349225 & 101510625677235 & 101510625677235 & 147651856584014 & 147651856584014 \\
\hline 1243 & 13773021513812 & 151503245506811 & 151503245506811 & 220368311165802 & 220368311165802 \\
\hline 1267 & 20477101283532 & 225248103749002 & 225248103749002 & 327633661514469 & 327633661514469 \\
\hline 1291 & 30330817057006 & 333638999586028 & 333638999586028 & 485293022305906 & 485293022305906 \\
\hline 1315 & 44763186057643 & 492395032232149 & 492395032232149 & 716211038247564 & 716211038247564 \\
\hline 1339 & 65829766710070 & 724127452040606 & 724127452040606 & 1053276194195726 & 1053276194195726 \\
\hline 1363 & 96478169516795 & 1061259841697878 & 1061259841697878 & 1543650799851638 & 1543650799851638 \\
\hline 1387 & 140922592375703 & 1550148543933051 & 1550148543933051 & 2254761371678042 & 2254761371678042 \\
\hline 1411 & 205170323111458 & 2256873521699226 & 2256873521699226 & 3282725299689321 & 3282725299689321 \\
\hline 1435 & 297760377206316 & 3275364187275260 & 3275364187275260 & 4764165877605326 & 4764165877605326 \\
\hline 1459 & 430796502686742 & 4738761484014951 & 4738761484014951 & 6892744232195987 & 6892744232195987 \\
\hline 1483 & 621388898570251 & 6835277940289662 & 6835277940289662 & 9942222151968754 & 9942222151968754 \\
\hline 1507 & 893660568325681 & 9830266182559709 & 9830266182559709 & 14298569361270602 & 14298569361270602 \\
\hline 1531 & 1281533705334823 & 14096870841750772 & 14096870841750772 & 20504538963932962 & 20504538963932962 \\
\hline 1555 & 1832589066704092 & 20158479636409529 & 20158479636409529 & 29321425454783979 & 29321425454783979 \\
\hline 1579 & 2613400611330406 & 28747406838047288 & 28747406838047288 & 41814409315423788 & 41814409315423788 \\
\hline 1603 & 3716891022406435 & 40885801111701610 & 40885801111701610 & 59470256913313545 & 59470256913313545 \\
\hline 1627 & 5272453300096449 & 57996986464667060 & 57996986464667060 & 84359252145641326 & 84359252145641326 \\
\hline 1651 & 7459846110139069 & 82058307013061490 & 82058307013061490 & 119357538538247375 & 119357538538247375 \\
\hline 1675 & 10528232420636209 & 115810556863115569 & 115810556863115569 & 168451717806641647 & 168451717806641647 \\
\hline 1699 & 14822207710919004 & 163044284544165030 & 163044284544165030 & 237155324477163395 & 237155324477163395 \\
\hline 1723 & 20817315567358978 & 228990471562943319 & 228990471562943319 & 333077047765997253 & 333077047765997253 \\
\hline 1747 & 29168407625477909 & 320852483499759068 & 320852483499759068 & 466694523558355316 & 466694523558355316 \\
\hline 1771 & 40775370225169032 & 448529072932153077 & 448529072932153077 & 652405921777788429 & 652405921777788429 \\
\hline 1795 & 56872276713083413 & 625595043298945690 & 625595043298945690 & 909956429556887554 & 909956429556887554 \\
\hline 1819 & 79148095494043006 & 870629051078933309 & 870629051078933309 & 1266369525369451301 & 1266369525369451301 \\
\hline 1843 & 109909812683600080 & 1209007938767976703 & 1209007938767976703 & 1758557005937509432 & 1758557005937509432 \\
\hline 1867 & 152302485633902688 & 1675327342846829765 & 1675327342846829765 & 2436839766599162279 & 2436839766599162279 \\
\hline 1891 & 210605556865399279 & 2316661124493547173 & 2316661124493547173 & 3369688914010001224 & 3369688914010001224 \\
\hline 1915 & 290631176879715729 & 3196942946893625371 & 3196942946893625371 & 4650098825202784367 & 4650098825202784367 \\
\hline 1939 & 400258719166874651 & 4402845909393277271 & 4402845909393277271 & 6404139512372754278 & 6404139512372754278 \\
\hline 1963 & 550150869077258932 & 6051659561542349276 & 6051659561542349276 & 8802413898544613901 & 8802413898544613901 \\
\hline 1987 & 754711357815895642 & 8301824934007779454 & 8301824934007779454 & 12075381732915932852 & 12075381732915932852 \\
\hline 2011 & 1033363835278555220 & 11367002190347074914 & 11367002190347074914 & 16533821355238048767 & 16533821355238048767 \\
\hline 2035 & 1412256805146747435 & 15534824853948167213 & 15534824853948167213 & 22596108893118543439 & 22596108893118543439 \\
\hline 2059 & 1926533042028023191 & 21191863465442002954 & 21191863465442002954 & 30824528659900088909 & 30824528659900088909 \\
\hline 2083 & 2623345678439652253 & 28856802459153067204 & 28856802459153067204 & 41973530869649280580 & 41973530869649280580 \\
\hline 2107 & 3565860601400413225 & 39224466619703622922 & 39224466619703622922 & 57053769605360276336 & 57053769605360276336 \\
\hline 2131 & 4838559710474916833 & 53224156810243842644 & 53224156810243842644 & 77416955387499037845 & 77416955387499037845 \\
\hline 2155 & 6554257603147504112 & 72096833640381599416 & 72096833640381599416 & 104868121627158155768 & 104868121627158155768 \\
\hline 2179 & 8863371782121759020 & 97497089596648487456 & 97497089596648487456 & 141813948540918808370 & 141813948540918808370 \\
\hline 2203 & 11966152827447202656 & 131627681109732904436 & 131627681109732904436 & 191458445207879722486 & 191458445207879722486 \\
\hline 2227 & 16128796948623520740 & 177416766425737654861 & 177416766425737654861 & 258060751214233038172 & 258060751214233038172 \\
\hline 2251 & 21704644236610653731 & 238751086613304015784 & 238751086613304015784 & 347274307743692860669 & 347274307743692860669 \\
\hline 2275 & 29162029847290462732 & 320782328307975295104 & 320782328307975295104 & 466592477605502723278 & 466592477605502723278 \\
\hline 2299 & 39120827378017906356 & 430329101172286761258 & 430329101172286761258 & 625933237991633696014 & 625933237991633696014 \\
\hline
\end{tabular}

Table 6. Multiplicity generating functions $f_{\mathrm{dP}_{8}, \chi}^{(1)}$, part 3 . 


\begin{tabular}{|c|c|c|c|c|c|}
\hline $24 d$ & 45 & 54 & 55 & $55^{\prime}$ & $55^{\prime \prime}$ \\
\hline 1171 & 184203114462622 & 221043710022343 & 225137084071984 & 225137106948035 & 225137106948035 \\
\hline 1195 & 277140803111755 & 332568997319530 & 338727716741557 & 338727688687159 & 338727688687159 \\
\hline 1219 & 415270838158915 & 498324964525296 & 507553162674521 & 507553197014225 & 507553197014225 \\
\hline 1243 & 619785885692343 & 743743113342304 & 757516185209007 & 757516143250948 & 757516143250948 \\
\hline 1267 & 921469660027838 & 1105763530523634 & 1126240570403383 & 1126240621565028 & 1126240621565028 \\
\hline 1291 & 1364886640954779 & 1637864043787277 & 1668194935551870 & 1668194873292078 & 1668194873292078 \\
\hline 1315 & 2014343526237095 & 2417212140959443 & 2461975236268978 & 2461975311902653 & 2461975311902653 \\
\hline 1339 & 2962339318825550 & 3554807292476658 & 3620637169265217 & 3620637077537819 & 3620637077537819 \\
\hline 1363 & 4341517847042590 & 5209821283094568 & 5306299319337420 & 5306299430386979 & 5306299430386979 \\
\hline 1387 & 6341516391505723 & 7609819831217510 & 7750742584630229 & 7750742450426761 & 7750742450426761 \\
\hline 1411 & 9232664864481335 & 11079197642782316 & 11284367771598830 & 11284367933524970 & 11284367933524970 \\
\hline 1435 & 13399216579858759 & 16079060129761174 & 16376820741075612 & 16376820545992293 & 16376820545992293 \\
\hline 1459 & 19385843094557620 & 23263011432427915 & 23693807653507039 & 23693807888163762 & 23693807888163762 \\
\hline 1483 & 27962499872265004 & 33555000184510544 & 34176389421242631 & 34176389139433773 & 34176389139433773 \\
\hline 1507 & 40214726244543434 & 48257671087653672 & 49151331250463847 & 49151331588413804 & 49151331588413804 \\
\hline 1531 & 57669015937475566 & 69202819611374765 & 70484353802379119 & 70484353397662854 & 70484353397662854 \\
\hline 1555 & 82466508969735861 & 98959810182387002 & 100792398668290829 & 100792399152265772 & 100792399152265772 \\
\hline 1579 & 117603026344789245 & 141123632306950527 & 143737033611788997 & 143737033033854903 & 143737033033854903 \\
\hline 1603 & 167260097396709360 & 200712116050021046 & 204429006245393365 & 204429006934618721 & 204429006934618721 \\
\hline 1627 & 237260396863613020 & 284712477220607098 & 289984931505798416 & 289984930684900175 & 289984930684900175 \\
\hline 1651 & 335693076895668315 & 402831691102628332 & 410291536040979311 & 410291537017434228 & 410291537017434228 \\
\hline 1675 & 473770456621652456 & 568524549339514438 & 579052783152120837 & 579052781992128638 & 579052781992128638 \\
\hline 1699 & 666999349746211386 & 800399218042669045 & 815221424102015049 & 815221425478366554 & 815221425478366554 \\
\hline 1723 & 936779197250978703 & 1124135038657868408 & 1144952356182632432 & 1144952354551480412 & 1144952354551480412 \\
\hline 1747 & 1312578347025836554 & 1575094014116214816 & 1604262419424649864 & 1604262421355471132 & 1604262421355471132 \\
\hline 1771 & 1834891655568344765 & 2201869989419897616 & 2242645362384455501 & 2242645360101610419 & 2242645360101610419 \\
\hline 1795 & 2559252457456586760 & 3071102945711666490 & 3127975219189577966 & 3127975221885611857 & 3127975221885611857 \\
\hline 1819 & 3561664290897644899 & 4273997152896785912 & 4353145252207517405 & 4353145249026968359 & 4353145249026968359 \\
\hline 1843 & 4945941578258894303 & 5935129889410954110 & 6045039697596848087 & 6045039701344867210 & 6045039701344867210 \\
\hline 1867 & 6853611844665722902 & 8224334218891965090 & 8376636709820185138 & 8376636705408223928 & 8376636705408223928 \\
\hline 1891 & 9477250069357366762 & 11372700077007117472 & 11583305627646850394 & 11583305632834988246 & 11583305632834988246 \\
\hline 1915 & 13078402947401857383 & 15694083544192819487 & 15984714728386214908 & 15984714722291519041 & 15984714722291519041 \\
\hline 1939 & 18011642376763874898 & 21613970843532086552 & 22014229554115942440 & 22014229561268362549 & 22014229561268362549 \\
\hline 1963 & 24756789091754815561 & 29708146920173901712 & 30258297799313493059 & 30258297790928171326 & 30258297790928171326 \\
\hline 1987 & 33962011121364327166 & 40754413333847397958 & 41509124679877898687 & 41509124689699169994 & 41509124689699169994 \\
\hline 2011 & 46501372564484998594 & 55801647091170275277 & 56835010940239663338 & 56835010928747353467 & 56835010928747353467 \\
\hline 2035 & 63551556258539592867 & 76261867494133421282 & 77674124283157975065 & 77674124296593010152 & 77674124296593010152 \\
\hline 2059 & 86693986859883218954 & 104032784250684215406 & 105959317311542087994 & 105959317295850490724 & 105959317295850490724 \\
\hline 2083 & 118050555566317547234 & 141660666657604661458 & 144284012314071615021 & 144284012332382347583 & 144284012332382347583 \\
\hline 2107 & 160463727020416092994 & 192556472450127762662 & 196122333077146178381 & 196122333055797906898 & 196122333055797906898 \\
\hline 2131 & 217735187021112290780 & 261282224395485799571 & 266120784076119006487 & 266120784100986919412 & 266120784100986919412 \\
\hline 2155 & 294941592083627118072 & 353929910535079510346 & 360484168172958312466 & 360484168144015486852 & 360484168144015486852 \\
\hline 2179 & 398851730262924276402 & 478622076275134635183 & 487485448016868002166 & 487485448050525203783 & 487485448050525203783 \\
\hline 2203 & 538476877156922474149 & 646172252635225208162 & 658138405509601252323 & 658138405470493978887 & 658138405470493978887 \\
\hline 2227 & 725795862778692020557 & 870955035279941337060 & 887083832174081304741 & 887083832219484026698 & 887083832219484026698 \\
\hline 2251 & 976708990542309495285 & 1172050788713993804393 & 1193755433013807220005 & 1193755432961138141154 & 1193755432961138141154 \\
\hline 2275 & 1312291343250192222140 & 1574749611826956036768 & 1603911641600986492613 & 1603911641662036771525 & 1603911641662036771525 \\
\hline 2299 & 1760437231869163874840 & 2112524678327840972437 & 2151645505790711677538 & 2151645505720001135999 & 2151645505720001135999 \\
\hline
\end{tabular}

Table 7. Multiplicity generating functions $f_{\mathrm{dP}_{8}, \chi}^{(1)}$, part 4 . 


\begin{tabular}{|c|c|c|c|c|c|}
\hline $24 d$ & 66 & 99 & 120 & 144 & 176 \\
\hline 1171 & 270164546681156 & 405246797027805 & 491208274997242 & 589449911767971 & 720438760605286 \\
\hline 1195 & 406473203907733 & 609709834093878 & 739042178780400 & 886850636998028 & 1083928581424780 \\
\hline 1219 & 609063863910983 & 913595761484332 & 1107388855916070 & 1328866599517240 & 1624170257738622 \\
\hline 1243 & 909019338414137 & 1363529049387265 & 1652762418194392 & 1983314935496409 & 2424051624958728 \\
\hline 1267 & 1351488786694107 & 2027233129147441 & 2457252358138100 & 2948702788864913 & 3603970030037952 \\
\hline 1291 & 2001833798159548 & 3002750759449688 & 3639697792134294 & 4367637400215692 & 5338223544445360 \\
\hline 1315 & 2954370434912214 & 4431555576448250 & 5371582636389448 & 6445899103301566 & 7878321058779942 \\
\hline 1339 & 4344764419512895 & 6517146721381044 & 7899571638612220 & 9479486039749018 & 11586038574903016 \\
\hline 1363 & 6367559405323185 & 9551338996863518 & 11577380777243530 & 13892856843630246 & 16980158265658175 \\
\hline 1387 & 9300890833306805 & 13951336383760152 & 16910710557156234 & 20292852776170360 & 24802375734252748 \\
\hline 1411 & 13541241649561517 & 20311862312968942 & 24620439421902434 & 29544527176781278 & 36109977517072127 \\
\hline 1435 & 19652184499179137 & 29478276943727693 & 35731244472881150 & 42877493523182575 & 52405825590524819 \\
\hline 1459 & 28432569653735348 & 42648854245377598 & 51695581273866940 & 62034697341246448 & 75820185430196005 \\
\hline 1483 & 41011666741538202 & 61517500394937483 & 74566666700591406 & 89480000266235449 & 109364445021070861 \\
\hline 1507 & 58981598176550861 & 88472396926681358 & 107239269534589732 & 128687123170645012 & 157284261352456668 \\
\hline 1531 & 84581223753771591 & 126871836034520310 & 153784043041385876 & 184540851973883398 & 225549930549289886 \\
\hline 1555 & 120950879369401488 & 181426318571322984 & 219910689938939870 & 263892827539670898 & 322535677674888309 \\
\hline 1579 & 172484439178360861 & 258726659345247778 & 313608071022950170 & 376329685689204628 & 459958505244752664 \\
\hline 1603 & 245314808873442099 & 367972212619696564 & 446026925474876674 & 535232310019095576 & 654172822742952752 \\
\hline 1627 & 347981916164512698 & 521972875069314411 & 632694392728415992 & 759233271930954787 & 927951777535669872 \\
\hline 1651 & 492349845202179646 & 738524766826508033 & 895181537085930972 & 1074217843720999295 & 1312932919235178135 \\
\hline 1675 & 694863337463226605 & 1042295007353137208 & 1263387885874730544 & 1516065463978597964 & 1852968901447872354 \\
\hline 1699 & 978265711674229209 & 1467398566137307066 & 1778664930818030794 & 2134397915880732844 & 2608708562632879662 \\
\hline 1723 & 1373942824157073997 & 2060914237866252831 & 2498077861510042094 & 2997693435115570067 & 3663847533257402807 \\
\hline 1747 & 1925114907172125283 & 2887672358825009115 & 3500208922832935414 & 4200250705856191083 & 5133639749883610819 \\
\hline 1771 & 2691174430294308510 & 4036761647727631051 & 4893044417887901864 & 5871653303292074233 & 7176465150500213001 \\
\hline 1795 & 3753570268419902966 & 5630355399933081775 & 6824673216288466950 & 8189607857387351047 & 10009520712187446446 \\
\hline 1819 & 5223774296289292241 & 7835661447611118530 & 9497771446641673624 & 11397325738516222932 & 13930064794343989332 \\
\hline 1843 & 7254047644610345604 & 10881071463172141658 & 13189177537019635416 & 15827013041425604314 & 19344127047303818283 \\
\hline 1867 & 10051964042960644068 & 15077946068852005519 & 18276298258323001986 & 21931557913514503889 & 26805237453771981804 \\
\hline 1891 & 13899966763554443537 & 20849950140138817382 & 25272666844712164358 & 30327200209506516810 & 37066578029225470889 \\
\hline 1915 & 19181657661871572164 & 28772486498908357454 & 34875741201188675430 & 41850889446302706172 & 51151087106459775710 \\
\hline 1939 & 26417075479244374467 & 39625613211712943497 & 48031046328498284852 & 57637255588472376133 & 70445534601774047231 \\
\hline 1963 & 36309957342408061267 & 54464936021991049526 & 66018104255873659084 & 79221725113760100204 & 96826552924265854344 \\
\hline 1987 & 49810949635492675761 & 74716424443426330769 & 90565362977197226288 & 108678435564780334215 & 132829199014898120022 \\
\hline 2011 & 68202013105303745330 & 102303019669446105608 & 124003660187280231114 & 148804392233925001344 & 181872034962788778272 \\
\hline 2035 & 93208949166662983780 & 139813423736550819498 & 169470816671544273222 & 203364979995109897828 & 248557197759850986121 \\
\hline 2059 & 127151180742462470212 & 190726771129397283986 & 231183964980593334826 & 277420757989266397324 & 339069815334173156022 \\
\hline 2083 & 173140814813508567478 & 259711222201949509299 & 314801481485761998530 & 377761777768258741081 & 461708839478260064519 \\
\hline 2107 & 235346799649883728800 & 353020199496161859438 & 427903272082932960552 & 513483926516604492932 & 627591465761482246451 \\
\hline 2131 & 319344940941071942661 & 479017411386756381538 & 580627165356451864750 & 696752598407849494756 & 851586509143058978448 \\
\hline 2155 & 432581001749665552605 & 648871502653437926113 & 786510912261590705900 & 943813094737053749501 & 1153549338037678618915 \\
\hline 2179 & 584982537687562696957 & 877473806497670520373 & 1063604613989623332136 & 1276325536760630740267 & 1559953433788616761379 \\
\hline 2203 & 789766086533298317643 & 1184649129839076522389 & 1435938339137237812658 & 1723126006995973066501 & 2106042897474304114574 \\
\hline 2227 & 1064500598699704522077 & 1596750898004149803311 & 1935455634015967754356 & 2322546760782826588291 & 2838668263138648397534 \\
\hline 2251 & 1432506519511239076275 & 2148759779319506061861 & 2604557308183097498046 & 3125468769861864014025 & 3820017385433520294132 \\
\hline 2275 & 1924693970043272902043 & 2887040955003888251120 & 3499443581919069498760 & 4199332298254045573934 & 5132517253367370460268 \\
\hline 2299 & 2581974606807435465725 & 3872961910281857647683 & 4694499285078708148956 & 5633399142151001036453 & 6885265618247405253843 \\
\hline
\end{tabular}

Table 8. Multiplicity generating functions $f_{\mathrm{dP}_{8}, \chi}^{(1)}$, part 5 . 
Open Access. This article is distributed under the terms of the Creative Commons Attribution License (CC-BY 4.0), which permits any use, distribution and reproduction in any medium, provided the original author(s) and source are credited.

\section{References}

[1] A. Dabholkar, S. Murthy and D. Zagier, Quantum Black Holes, Wall Crossing and Mock Modular Forms, arXiv:1208.4074 [INSPIRE].

[2] T. Eguchi, H. Ooguri and Y. Tachikawa, Notes on the K3 Surface and the Mathieu Group M24, Exper. Math. 20 (2011) 91.

[3] M.C.N. Cheng, J.F.R. Duncan and J.A. Harvey, Umbral Moonshine and the Niemeier Lattices, Res. Math. Sci. 1 (2014) 3 [arXiv:1307.5793] [InSPIRE].

[4] M.C.N. Cheng, J.F.R. Duncan and J.A. Harvey, Weight One Jacobi Forms and Umbral Moonshine, J. Phys. A 51 (2018) 104002 [arXiv:1703.03968] [InSPIRE].

[5] N.-P. Skoruppa, Developments in the theory of Jacobi forms, in Automorphic functions and their applications, Khabarovsk (1988), pp. 167-185.

[6] N.-P. Skoruppa, Explicit formulas for the Fourier coefficients of Jacobi and elliptic modular forms, Invent. Math. 102 (1990) 501.

[7] J.A. Harvey and B.C. Rayhaun, Traces of Singular Moduli and Moonshine for the Thompson Group, Commun. Num. Theor. Phys. 10 (2016) 23 [arXiv:1504.08179] [InSPIRE].

[8] J.F.R. Duncan, J.A. Harvey and B.C. Rayhaun, Skew-Holomorphic Jacobi Forms and Moonshine, in preparation.

[9] J.M. Maldacena, A. Strominger and E. Witten, Black hole entropy in M-theory, JHEP 12 (1997) 002 [hep-th/9711053] [INSPIRE].

[10] D. Gaiotto, A. Strominger and X. Yin, The M5-Brane Elliptic Genus: Modularity and BPS States, JHEP 08 (2007) 070 [hep-th/0607010] [INSPIRE].

[11] J. de Boer, M.C.N. Cheng, R. Dijkgraaf, J. Manschot and E. Verlinde, A Farey Tail for Attractor Black Holes, JHEP 11 (2006) 024 [hep-th/0608059] [INSPIRE].

[12] F. Denef and G.W. Moore, Split states, entropy enigmas, holes and halos, JHEP 11 (2011) 129 [hep-th/0702146] [INSPIRE].

[13] J. Manschot and G.W. Moore, A Modern Farey Tail, Commun. Num. Theor. Phys. 4 (2010) 103 [arXiv:0712.0573] [INSPIRE].

[14] M. Alim, B. Haghighat, M. Hecht, A. Klemm, M. Rauch and T. Wotschke, Wall-crossing holomorphic anomaly and mock modularity of multiple M5-branes, Commun. Math. Phys. 339 (2015) 773 [arXiv:1012.1608] [INSPIRE].

[15] M.C.N. Cheng and J.F.R. Duncan, Optimal Mock Jacobi Theta Functions, arXiv: 1605.04480 [INSPIRE].

[16] M.C.N. Cheng and J.F.R. Duncan, Meromorphic Jacobi Forms of Half-Integral Index and Umbral Moonshine Modules, arXiv:1707.01336 [INSPIRE].

[17] N.-P. Skoruppa and D. Zagier, Jacobi forms and a certain space of modular forms, Invent. Math. 94 (1988) 113. 
[18] N.-P. Skoruppa, Heegner cycles, modular forms and Jacobi forms, J. Théor. Nombres Bordeaux 3 (1991) 93.

[19] J. Duncan, M. Mertens and K. Ono, O'Nan moonshine and arithmetic, arXiv:1702.03516.

[20] G.W. Moore, Arithmetic and attractors, hep-th/9807087 [INSPIRE].

[21] P. Di Francesco, P. Mathieu and D. Senechal, Conformal Field Theory, Springer-Verlag, New York (1997).

[22] G.W. Moore and N. Seiberg, Naturality in Conformal Field Theory, Nucl. Phys. B 313 (1989) 16 [INSPIRE].

[23] A. Dabholkar and J.A. Harvey, Nonrenormalization of the Superstring Tension, Phys. Rev. Lett. 63 (1989) 478 [INSPIRE].

[24] V.V. Nikulin, Integral Symmetric Bilinear Forms and Some of their Applications, Math. USSR Izv. 14 (1980) 103 [Izv. Akad. Nauk SSSR Ser. Mat. 43 (1979) 111].

[25] J.H. Conway and N.J.A. Sloane. Sphere packings, lattices and groups, vol. 290, Springer Science \& Business Media (2013).

[26] W. Ebeling, Lattices and Codes. A course partially based on lectures by Friedrich Hirzebruch, Advanced Lectures in Mathematics, Springer Spektrum (2013).

[27] R. Minasian, G.W. Moore and D. Tsimpis, Calabi-Yau black holes and $(0,4) \sigma$-models, Commun. Math. Phys. 209 (2000) 325 [hep-th/9904217] [INSPIRE].

[28] L. Göttsche, The Betti numbers of the Hilbert schemes of points on a smooth projective surface, Math. Ann. 286 (1990) 193.

[29] D. Zagier, Nombres de classes et formes modulaires de poids 3/2, Sém. Théor. Nombres Bordeaux 4 (1975) 1.

[30] M. Cheng, J. Duncan and M. Mertens, Class Number Moonshine, in preparation.

[31] K. Yoshioka, The Betti numbers of the moduli space of stable sheaves of rank 2 on $\mathbb{P}^{2}$, J. Reine Angew. Math. 453 (1994) 193.

[32] K. Yoshioka, The Betti numbers of the moduli space of stable sheaves of rank 2 on a ruled surface, Math. Ann. 302 (1995) 519.

[33] C. Vafa and E. Witten, A Strong coupling test of S duality, Nucl. Phys. B 431 (1994) 3 [hep-th/9408074] [INSPIRE].

[34] R. Griess and A. Ryba, Finite simple groups which projectively embed in an exceptional Lie group are classified!, Bull. Am. Math. Soc. 36 (1999) 75.

[35] S. Kachru and A. Tripathy, Black Holes and Hurwitz Class Numbers, Int. J. Mod. Phys. D 26 (2017) 1742003 [arXiv: 1705.06295] [INSPIRE].

[36] J.A. Minahan, D. Nemeschansky, C. Vafa and N.P. Warner, E strings and $N=4$ topological Yang-Mills theories, Nucl. Phys. B 527 (1998) 581 [hep-th/9802168] [INSPIRE].

[37] M.C.N. Cheng and J.F.R. Duncan, Rademacher Sums and Rademacher Series, Contrib. Math. Comput. Sci. 8 (2014) 143 [arXiv:1210.3066] [INSPIRE]. 